\title{
Quark gap equation with non-Abelian Ball-Chiu vertex
}

\author{
A. C. Aguilar, ${ }^{1}$ J. C. Cardona, ${ }^{1}$ M. N. Ferreira, ${ }^{1}$ and J. Papavassiliou ${ }^{2}$ \\ ${ }^{1}$ University of Campinas-UNICAMP, Institute of Physics "Gleb Wataghin", \\ 13083-859 Campinas, São Paulo, Brazil \\ ${ }^{2}$ Department of Theoretical Physics and IFIC, University of Valencia and CSIC, E-46100 Valencia, Spain
}

(Received 16 April 2018; published 2 July 2018)

\begin{abstract}
The full quark-gluon vertex is a crucial ingredient for the dynamical generation of a constituent quark mass from the standard quark gap equation, and its nontransverse part may be determined exactly from the nonlinear Slavnov-Taylor identity that it satisfies. The resulting expression involves not only the quark propagator, but also the ghost dressing function and the quark-ghost kernel, and constitutes the non-abelian extension of the so-called "Ball-Chiu vertex," known from QED. In the present work we carry out a detailed study of the impact of this vertex on the gap equation and the quark masses generated from it, putting particular emphasis on the contributions directly related with the ghost sector of the theory, and especially the quark-ghost kernel. In particular, we set up and solve the coupled system of six equations that determine the four form factors of the latter kernel and the two typical Dirac structures composing the quark propagator. Due to the incomplete implementation of the multiplicative renormalizability at the level of the gap equation, the correct anomalous dimension of the quark mass is recovered through the inclusion of a certain function, whose ultraviolet behavior is fixed, but its infrared completion is unknown; three particular Ansätze for this function are considered, and their effect on the quark mass and the pion decay constant is explored. The main results of this study indicate that the numerical impact of the quark-ghost kernel is considerable; the transition from a tree-level kernel to the one computed here leads to a $20 \%$ increase in the value of the quark mass at the origin. Particularly interesting is the contribution of the fourth Ball-Chiu form factor, which, contrary to the Abelian case, is nonvanishing, and accounts for $10 \%$ of the total constituent quark mass.
\end{abstract}

DOI: $10.1103 /$ PhysRevD.98.014002

\section{INTRODUCTION}

The dynamical breaking of chiral symmetry and the generation of a constituent mass for the quarks represent two of the most important emergent phenomena in QCD, and the detailed study of the nonperturbative dynamics associated with them has been the focal point of countless articles spanning several decades [1-23]. One of the standard frameworks employed in this pursuit is the socalled "quark gap-equation," namely the Schwinger-Dyson equation (SDE) $[10,13]$ that controls the evolution of the quark propagator $S(p)$. This special integral equation is particularly sensitive to the ingredients that compose its kernel, and in particular on the details of the fully-dressed quark-gluon vertex $\Gamma_{\mu}\left(q, p_{2},-p_{1}\right)$ [10]. This latter threepoint function is built out of twelve linearly independent tensorial structures [24-27], and the determination of

Published by the American Physical Society under the terms of the Creative Commons Attribution 4.0 International license. Further distribution of this work must maintain attribution to the author(s) and the published article's title, journal citation, and DOI. Funded by SCOAP . the nonperturbative behavior of the corresponding form-factors represents a major challenge for the contemporary field-theoretic formalisms, both continuous and discrete $[18,23,28-49]$.

The quark-gluon vertex $\Gamma_{\mu}$ satisfies a nonlinear SlavnovTaylor identity (STI), given by $q^{\mu} \Gamma_{\mu}\left(q, p_{2},-p_{1}\right)=$ $F(q)\left[S^{-1}\left(p_{1}\right) H\left(q, p_{2},-p_{1}\right)-\bar{H}\left(-q, p_{1},-p_{2}\right) S^{-1}\left(p_{2}\right)\right]$, where $F(q)$ is the dressing function of the ghost propagator, and $H$ is the so-called quark-ghost kernel, which consists of four linearly independent tensorial structures, and $S^{-1}(p)=A(p) \not p-B(p)$. When the ghost sector is switched off (i.e., $F=H=1$ ), the above STI reduces to the standard Ward-Takahashi identity of QED. It is common practice to decompose $\Gamma_{\mu}$ into two parts, $\Gamma_{\mu}=\Gamma_{\mu}^{\mathrm{STI}}+\Gamma_{\mu}^{\mathrm{T}}$, where $\Gamma_{\mu}^{\mathrm{STI}}$ saturates the above STI, while $\Gamma_{\mu}^{\mathrm{STI}}$ denotes the transverse (automatically conserved) part, (i.e., $q^{\mu} \Gamma_{\mu}^{\mathrm{T}}=0$ ). Then, it turns out that the four form factors comprising $\Gamma_{\mu}^{\mathrm{STI}}$, to be denoted by $L_{i}$, may be expressed entirely in terms of combinations involving $A, B$, and the form factors of $H$. The $\Gamma_{\mu}^{\text {STI }}$ obtained from the Abelianized version of the STI (setting $F=H=1$ ) is known in the literature as the "BallChiu" vertex [24], and will be denoted by $\Gamma_{\mu}^{\mathrm{BC}}$. In order to 
establish a clear distinction between $\Gamma_{\mu}^{\mathrm{BC}}$ and the full $\Gamma_{\mu}^{\mathrm{STI}}$, which includes, at least in principle, all ghost related contributions (and, in particular, those from $H$ ), we will denominate the latter as the "non-Abelian Ball-Chiu vertex".

Since the form factors of $H$, to be denoted by $X_{i}$, constitute an indispensable ingredient for the complete determination of $\Gamma_{\mu}^{\mathrm{STI}}$, in a recent work [40] a SDE-based procedure was developed for their dynamical determination. Specifically, the skeleton expansion of $H$ was truncated at its "one-loop-dressed" level, and the four $X_{i}$ were determined by means of appropriate projections, for arbitrary values of Euclidean momenta. As a result, one obtained approximate expressions for the form factors of $\Gamma_{\mu}^{\text {STI }}$, which receive nontrivial contributions from the kernel $H$, whose numerical impact is quite considerable. In particular, not only is the difference between $\Gamma_{\mu}^{\mathrm{STI}}$ and $\Gamma_{\mu}^{\mathrm{BC}}$ particularly pronounced, but a considerable difference is found also between $\Gamma_{\mu}^{\mathrm{STI}}$ and the "minimally non-Abelianized" Ball-Chiu vertex, obtained by multiplying $\Gamma_{\mu}^{\mathrm{BC}}$ by $F(q)$; we denote this latter vertex by $\Gamma_{\mu}^{\mathrm{FBC}}=F(q) \Gamma_{\mu}^{\mathrm{BC}}[12,40]$. Note that the resulting form factors of $\Gamma_{\mu}^{\mathrm{STI}}\left(q, p_{2},-p_{1}\right)$ display a completely nontrivial dependence on three kinematic variables, chosen to be the moduli of two of the incoming momenta, $p_{1}$ and $p_{2}$, and the angle $\theta$ between them.

Given that $\Gamma_{\mu}$ is known to be particularly relevant for the studies of the phenomena controlled by the gap equation, it is natural to explore the impact that the $\Gamma_{\mu}^{\mathrm{STI}}$ constructed in [40] might have on dynamical chiral symmetry breaking and quark mass generation. The purpose of the present work is to carry out a detailed quantitative study of this particular question, adding, at the same time, an extra layer of technical complexity to the considerations presented so far. Specifically, in the analysis of [40,50], $S(p)$ was essentially treated as an "external" quantity: the corresponding $A$ and $B$ used for the evaluation of the $X_{i}$ were obtained from solving a gap equation containing a simplified version of $\Gamma_{\mu}^{\mathrm{STI}}$. It is clear, however, that the self-consistent treatment of this problem requires the solution of a coupled system of several dynamical equations, given that $S(p)$ enters in the integrals that determine the form factors of $H$, which, in turn, enter through $\Gamma_{\mu}^{\mathrm{STI}}$ in the gap equation that determines $S(p)$. Therefore, in the analysis presented here, we will consider the intertwined dynamics produced by a system involving six coupled equations (four determining the $X_{i}$, and two the $A$ and $B$ ).

There are two important issues related to our analysis that need to be emphasized at this point. First, the gap equation is studied in the chiral limit, i.e., no "current" mass, $m_{0}$, is added to the corresponding equations [see, for example, Eq. (2.2)]. Second, the external ingredients used (see subsection III A) are obtained from "quenched" lattice simulations; this simplification affects both the gluon

\footnotetext{
${ }^{1}$ In what follows we will use the terms " $\Gamma_{\mu}^{\text {STI" }}$ and "nonAbelian Ball-Chiu vertex" interchangeably.
}

propagator and the ghost dressing function, and, indirectly, the form factors of the quark-gluon vertex, and, eventually, the gap equation itself. Unquenching effects have been taken into account in the context of other approaches [29,38,51-53], and can also be treated within our formalism, along the lines presented in [54]. Such a study, however, lies beyond the main scope of the present work, which focuses on the impact that the fully non-Abelianized Ball-Chiu vertex has on the gap equation.

The main findings of our study may be summarized as follows:

(1) The dynamical quark masses, $\mathcal{M}(p)$, generated with $\Gamma_{\mu}^{\mathrm{STI}}$ are always higher than those obtained with the $\Gamma_{\mu}^{\mathrm{BC}}$. The precise amount depends on the specific value of $\alpha_{s}$ employed in the numerical calculation, but, on the average, the impact of $H$ on $\mathcal{M}(0)$ is of the order of $20 \%$ for the cases where $\mathcal{M}(p)$ is around 300-350 MeV [see Fig. 3]. The quark wave functions follow a similar pattern, with $A^{-1}(p)$ always larger than $A_{\mathrm{FBC}}^{-1}(p)$ [Fig. 3].

(2) The results for the vertex form factors, $L_{i}$, obtained after solving the coupled system, display the same qualitative and quantitative behavior found in Ref. [40], where $A(p)$ and $B(p)$ were treated as external ingredients [see Fig. 5].

(3) The form factor $L_{1}$ is responsible for generating more than half of the value of $\mathcal{M}(0)(54 \%)$, whereas $L_{2}$, and $L_{3}$ provide $13 \%$ and $23 \%$ of the quark mass value at zero momentum, respectively. Particularly interesting is the considerable contribution originating from the inclusion of $L_{4}$, which is commonly neglected in the quark SDE studies, accounting for $10 \%$ of $\mathcal{M}(0)$.

(4) The pion constant decay, $f_{\pi}$, was used as a simple indicator of the impact that the inclusion of $H$ in the construction of $\Gamma_{\mu}^{\text {STI }}$ might have on physical quantities. Our study reveals that the final impact of $H$ is to increase by $10 \%$ of the value of $f_{\pi}$ [see Table III].

(5) All quark masses obtained may be fitted accurately by two very simple formulas, given by Eqs. (3.1) and (3.2), which, at large momenta, reproduce the wellknown power-law behavior expressed in Eq. (2.17) [see Fig. 9].

The article is organized as follows. In Sec. II we introduce the notation and set up the theoretical framework of this work, and review the general structure of the gap equation, together with the SDEs for the four form factors, $X_{i}$. In Sec. III we present the numerical treatment of the system of six coupled integral equations, formed by $A(p), B(p)$ and the four $X_{i}$. Finally, in Sec. IV we present our conclusions.

\section{THEORETICAL INGREDIENTS AND DERIVATION OF THE SYSTEM}

In this section we review all ingredients and concepts necessary for arriving at the system of integral equations that is diagrammatically depicted in Fig. 1. 


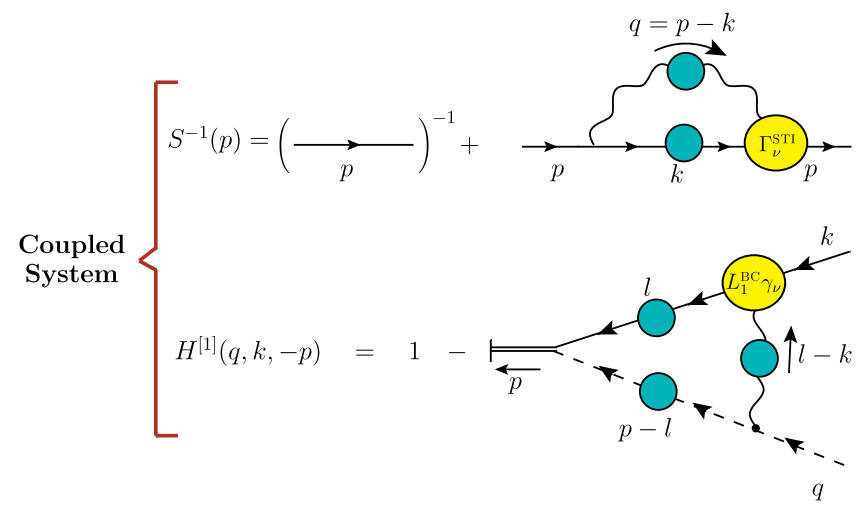

FIG. 1. The SDE for the quark propagator, $S(p)$ (top), and the quark-ghost scattering kernel at one-loop dressed approximation, $H^{[1]}(q, k,-p)$ (bottom). The quark-gluon vertex, $\Gamma_{\mu}^{\mathrm{STI}}$, couples $S(p)$ to $H^{[1]}(q, k,-p)$.

\section{A. Gap equation and quark-gluon vertex}

The full quark propagator can be written as

$$
S^{-1}(p)=A(p) \not p-B(p) \mathbb{I}=A(p)[\not p-\mathcal{M}(p) \mathbb{I}],
$$

where $A(p)$ and $B(p)$ are scalar functions whose ratio defines the dynamical quark mass function $\mathcal{M}(p)=$ $B(p) / A(p)$.

The momentum-dependence of $S(p)$, or, equivalently, of the functions $A(p)$ and $B(p)$, may be obtained from the quark gap equation, which, in its renormalized form, is given by

$S^{-1}(p)=Z_{\mathrm{F}} \not \supset-Z_{1} C_{\mathrm{F}} g^{2} \int_{k} \gamma_{\mu} S(k) \Gamma_{\nu}(q, k,-p) \Delta^{\mu \nu}(q)$,

where $C_{\mathrm{F}}$ is the Casimir eigenvalue for the fundamental representation, and we have introduced the compact notation for the integral measure

$$
\int_{k} \equiv \frac{\mu^{\epsilon}}{(2 \pi)^{d}} \int \mathrm{d}^{d} k
$$

with $\mu$ the 't Hooft mass, and $d=4-\epsilon$ the space-time dimension. In addition, $\Gamma_{\nu}(q, k,-p)$ is the full quark-gluon vertex, while $Z_{1}(\mu)$ and $Z_{\mathrm{F}}(\mu)$ are the vertex and the quark wave-function renormalization constants, respectively, and $\mu$ is the renormalization point. Moreover, in the Landau gauge, the full gluon propagator $\Delta_{\mu \nu}(q)$ is given by

$$
i \Delta_{\mu \nu}(q)=-i P_{\mu \nu}(q) \Delta(q) ; \quad P_{\mu \nu}(q)=g_{\mu \nu}-\frac{q_{\mu} q_{\nu}}{q^{2}} .
$$

Note finally that Eq. (2.2) is expressed in the chiral limit, since it contains no "current" quark mass $\left(m_{0}=0\right)$.

$\Gamma_{\mu}^{a}\left(q, p_{2},-p_{1}\right)$ may be cast in the form $\Gamma_{\mu}^{a}\left(q, p_{2},-p_{1}\right)=$ $g t^{a} \Gamma_{\mu}\left(q, p_{2},-p_{1}\right)$, where $g$ is the gauge coupling and $t^{a}$ are the $\mathrm{SU}(3)$ generators in the fundamental representation. $\Gamma_{\mu}\left(q, p_{2},-p_{1}\right)$ may be then separated into two distinct pieces,

$\Gamma_{\mu}\left(q, p_{2},-p_{1}\right)=\Gamma_{\mu}^{\mathrm{STI}}\left(q, p_{2},-p_{1}\right)+\Gamma_{\mu}^{\mathrm{T}}\left(q, p_{2},-p_{1}\right)$,

where $\Gamma_{\mu}^{\mathrm{T}}$ is transverse with respect to the momentum $q^{\mu}$ carried by the gluon,

$$
q^{\mu} \Gamma_{\mu}^{\mathrm{T}}\left(q, p_{2},-p_{1}\right)=0,
$$

while the first piece saturates the fundamental STI given by

$$
\begin{aligned}
q^{\mu} \Gamma_{\mu}^{\mathrm{STI}}\left(q, p_{2},-p_{1}\right)= & F(q)\left[S^{-1}\left(p_{1}\right) H\left(q, p_{2},-p_{1}\right)\right. \\
& \left.-\bar{H}\left(-q, p_{1},-p_{2}\right) S^{-1}\left(p_{2}\right)\right] .
\end{aligned}
$$

In the STI above, $F(q)$ is the dressing function of the full ghost propagator, $D(q)=i F(q) / q^{2}, H$ is the quark-ghost scattering kernel, shown diagrammatically in the second line of Fig. 1, while $\bar{H}$ is its "conjugate," whose relation to $H$ is explained in detail in [40]. Note that the color structure has been factored out, setting $H^{a}=-g t^{a} H$. The most general tensorial decompositions of $H$ and $\bar{H}$ read $[18,26,40]$

$$
\begin{aligned}
& H\left(q, p_{2},-p_{1}\right)=X_{0} \mathbb{I}+X_{1} \not p_{1}+X_{2} \not \not_{2}+X_{3} \tilde{\sigma}_{\mu \nu} p_{1}^{\mu} p_{2}^{\nu}, \\
& \bar{H}\left(-q, p_{1},-p_{2}\right)=\bar{X}_{0} \mathbb{I}+\bar{X}_{2} \not 1_{1}+\bar{X}_{1} \not \iota_{2}+\bar{X}_{3} \tilde{\sigma}_{\mu \nu} p_{1}^{\mu} p_{2}^{\nu} \text {, }
\end{aligned}
$$

where $\tilde{\sigma}_{\mu \nu} \equiv \frac{1}{2}\left[\gamma_{\mu}, \gamma_{\nu}\right]$, and we have introduced the compact notation for the form factors $X_{i}:=X_{i}\left(q^{2}, p_{2}^{2}, p_{1}^{2}\right)$ and $\bar{X}_{i}:=X_{i}\left(q^{2}, p_{1}^{2}, p_{2}^{2}\right)$. At tree-level, $X_{0}^{(0)}=\bar{X}_{0}^{(0)}=1$ and $X_{i}^{(0)}=\bar{X}_{i}^{(0)}=0$ for $i \geq 1$.

Next, we can write the most general Lorentz decomposition for $\Gamma_{\mu}^{\mathrm{STI}}$ as

$$
\begin{aligned}
\Gamma_{\mu}^{\mathrm{STI}}\left(q, p_{2},-p_{1}\right)= & L_{1} \gamma_{\mu}+L_{2}\left(\not p_{1}-\not p_{2}\right)\left(p_{1}-p_{2}\right)_{\mu} \\
& +L_{3}\left(p_{1}-p_{2}\right)_{\mu}+L_{4} \tilde{\sigma}_{\mu \nu}\left(p_{1}-p_{2}\right)^{\nu},
\end{aligned}
$$

where $L_{i}:=L_{i}\left(q^{2}, p_{2}^{2}, p_{1}^{2}\right)$ are the quark-gluon form factors.

It is clear that with the help of the Eq. (2.7) the form factors $L_{i}$, appearing in Eq. (2.9), may be expressed in terms of $A, B, F, X_{i}$, and $\bar{X}_{i}$. Factoring out the common $F(q)$, it is convenient to define $L_{i}:=F(q) \bar{L}_{i} / 2$, which leads us to 


$$
\begin{aligned}
\bar{L}_{1}= & A\left(p_{1}\right)\left[X_{0}-\left(p_{1}^{2}+p_{1} \cdot p_{2}\right) X_{3}\right]+A\left(p_{2}\right)\left[\bar{X}_{0}-\left(p_{2}^{2}+p_{1} \cdot p_{2}\right) \bar{X}_{3}\right]+B\left(p_{1}\right)\left(X_{2}-X_{1}\right)+B\left(p_{2}\right)\left(\bar{X}_{2}-\bar{X}_{1}\right) ; \\
\bar{L}_{2}= & \frac{1}{\left(p_{1}^{2}-p_{2}^{2}\right)}\left\{A\left(p_{1}\right)\left[X_{0}+\left(p_{1}^{2}-p_{1} \cdot p_{2}\right) X_{3}\right]-A\left(p_{2}\right)\left[\bar{X}_{0}+\left(p_{2}^{2}-p_{1} \cdot p_{2}\right) \bar{X}_{3}\right]\right\} \\
& -\frac{1}{\left(p_{1}^{2}-p_{2}^{2}\right)}\left\{B\left(p_{1}\right)\left(X_{1}+X_{2}\right)-B\left(p_{2}\right)\left(\bar{X}_{1}+\bar{X}_{2}\right)\right\} ; \\
\bar{L}_{3}= & \frac{2}{p_{1}^{2}-p_{2}^{2}}\left\{A\left(p_{1}\right)\left(p_{1}^{2} X_{1}+p_{1} \cdot p_{2} X_{2}\right)-A\left(p_{2}\right)\left(p_{2}^{2} \bar{X}_{1}+p_{1} \cdot p_{2} \bar{X}_{2}\right)-B\left(p_{1}\right) X_{0}+B\left(p_{2}\right) \bar{X}_{0}\right\} ; \\
\bar{L}_{4}= & A\left(p_{1}\right) X_{2}-A\left(p_{2}\right) \bar{X}_{2}-B\left(p_{1}\right) X_{3}+B\left(p_{2}\right) \bar{X}_{3} .
\end{aligned}
$$

Setting in Eq. (2.10) tree level values for the $X_{i}$ and $\bar{X}_{i}$, we obtain the form factors of the "minimally nonAbelianized" Ball-Chiu vertex, $\Gamma_{\mu}^{\mathrm{FBC}}=F(q) \Gamma_{\mu}^{\mathrm{BC}}$, given by $[12,18,40]$,

$$
\begin{aligned}
L_{1}^{\mathrm{FBC}} & =F(q) \frac{\left[A\left(p_{1}\right)+A\left(p_{2}\right)\right]}{2}, \\
L_{2}^{\mathrm{FBC}} & =F(q) \frac{\left[A\left(p_{1}\right)-A\left(p_{2}\right)\right]}{2\left(p_{1}^{2}-p_{2}^{2}\right)}, \\
L_{3}^{\mathrm{FBC}} & =-F(q) \frac{\left[B\left(p_{1}\right)-B\left(p_{2}\right)\right]}{p_{1}^{2}-p_{2}^{2}}, \\
L_{4}^{\mathrm{FBC}} & =0 .
\end{aligned}
$$

To proceed, we will insert into Eq. (2.2) the dressed quarkgluon vertex of Eq. (2.9), defining $p_{1}=p$ and $p_{2}=k$. It is important to keep in mind that the expressions for the form factors $L_{i}=F(q) \bar{L}_{i} / 2$ in terms of the $X_{i}$ are given by Eq. (2.10). Then, taking appropriate traces and applying the usual rules for going to Euclidean space [18], we derive the following expressions for the integral equations satisfied by $A(p)$ and $B(p)$,

$$
\begin{aligned}
p^{2} A(p) & =Z_{\mathrm{F}} p^{2}+Z_{1} 4 \pi C_{F} \alpha_{s} \int_{k} \mathcal{K}_{\mathrm{A}}(k, p) \Delta(q) F(q), \\
B(p) & =Z_{1} 4 \pi C_{F} \alpha_{s} \int_{k} \mathcal{K}_{\mathrm{B}}(k, p) \Delta(q) F(q),
\end{aligned}
$$

where $\alpha_{s}=g^{2}(\mu) / 4 \pi$, and we have introduced the kernels

$$
\begin{aligned}
\mathcal{K}_{\mathrm{A}}(k, p)= & \left\{\frac{3}{2}(k \cdot p) \bar{L}_{1}-\left[\bar{L}_{1}-\left(k^{2}+p^{2}\right) \bar{L}_{2}\right] h(p, k)\right\} \mathcal{Q}_{\mathrm{A}}(k) \\
& -\left\{\frac{3}{2} p \cdot(k+p) \bar{L}_{4}+\left(\bar{L}_{3}-\bar{L}_{4}\right) h(p, k)\right\} \mathcal{Q}_{\mathrm{B}}(k), \\
\mathcal{K}_{\mathrm{B}}(k, p)= & \left\{\frac{3}{2} k \cdot(k+p) \bar{L}_{4}-\left(\bar{L}_{3}+\bar{L}_{4}\right) h(p, k)\right\} \mathcal{Q}_{\mathrm{A}}(k) \\
& +\left\{\frac{3}{2} \bar{L}_{1}-2 h(p, k) \bar{L}_{2}\right\} \mathcal{Q}_{\mathrm{B}}(k),
\end{aligned}
$$

with the functions $h(p, k)$ and $\mathcal{Q}_{\mathrm{f}}(k)$ defined as

$$
h(p, k):=\frac{k^{2} p^{2}-(k \cdot p)^{2}}{q^{2}},
$$

and

$$
\mathcal{Q}_{\mathrm{f}}(k):=\frac{f(k)}{A^{2}(k) k^{2}+B^{2}(k)},
$$

where $f(k)$, appearing in the numerator of Eq. (2.15), can be either $A(k)$ or $B(k)$, depending on the index of $\mathcal{Q}$.

Clearly, the kernels $\mathcal{K}_{\mathrm{A}}$ and $\mathcal{K}_{\mathrm{B}}$ that enter in Eq. (2.12) depend on the various $\bar{L}_{i}$, which ultimately will couple the functions $A(p)$ and $B(p)$ with the four integral equations for the form factors $X_{i}$, to be presented in Eq. (2.26). However, as we explain in the next subsection, before proceeding to the solution of the system, an additional important approximation needs to be implemented at the level of Eq. (2.12).

\section{B. Approximate renormalization and the anomalous dimension of $\mathcal{M}(p)$}

It is relatively straightforward to establish that the STI of Eq. (2.7) imposes the relation $Z_{1}=Z_{\mathrm{c}}^{-1} Z_{\mathrm{F}} Z_{\mathrm{H}}$, where $Z_{\mathrm{c}}$ and $Z_{\mathrm{H}}$ are the renormalization constants of the ghost propagator and the quark-ghost scattering kernel, respectively. Now, we recall that, in the Landau gauge, both the quark self-energy and the quark-ghost kernel are finite at one-loop [55]; thus, at that order, $Z_{\mathrm{F}}=Z_{\mathrm{H}}=1$, and, therefore, $Z_{1}=Z_{\mathrm{c}}^{-1}$. Imposing the above relations on Eq. (2.12), we obtain the approximate version

$$
\begin{aligned}
p^{2} A(p) & =p^{2}+Z_{\mathrm{c}}^{-1} 4 \pi C_{F} \alpha_{s} \int_{k} \mathcal{K}_{\mathrm{A}}(k, p) \Delta(q) F(q), \\
B(p) & =Z_{\mathrm{c}}^{-1} 4 \pi C_{F} \alpha_{s} \int_{k} \mathcal{K}_{\mathrm{B}}(k, p) \Delta(q) F(q) .
\end{aligned}
$$

Even with these approximations, the presence of $Z_{c}^{-1}$ in front of the corresponding integrals complicates the analysis, especially in a nonperturbative setting [56-60]. It is wellknown that, in general, such multiplicative renormalization constants are instrumental for the systematic cancellation of overlapping divergences, whose complete implementation hinges, in addition, on the inclusion of crucial contributions stemming from the transverse parts of the vertices involved 

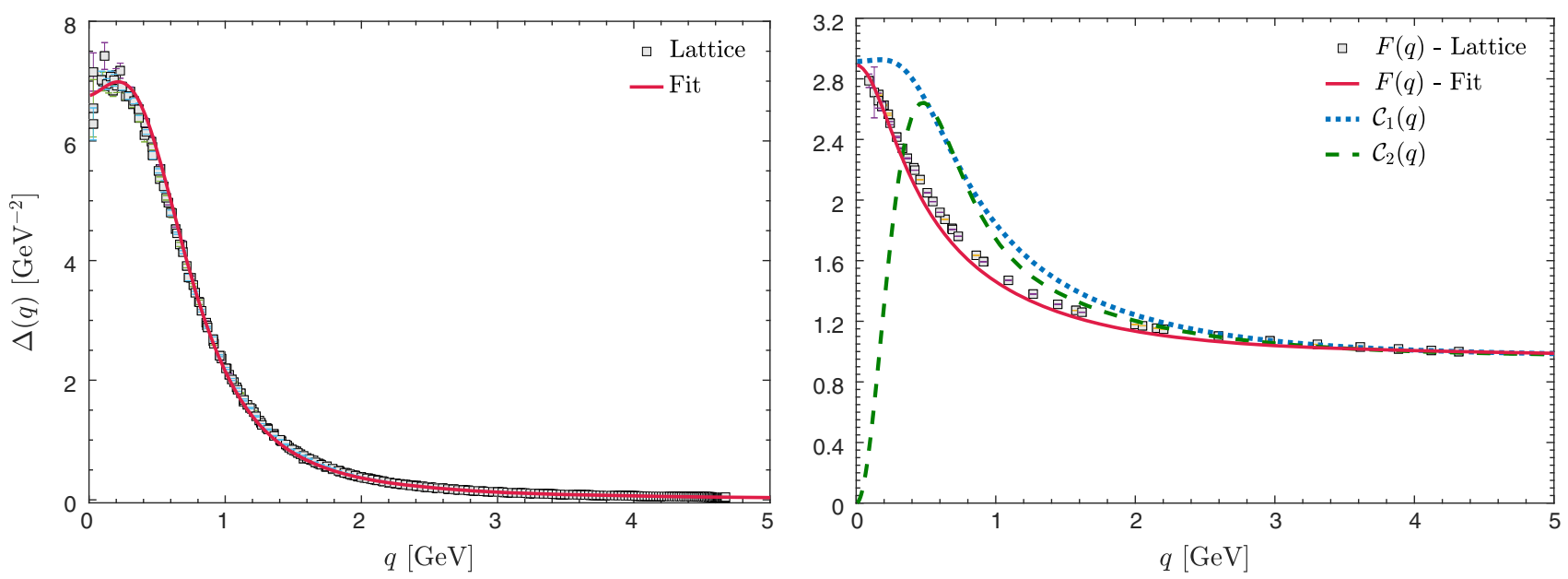

FIG. 2. The gluon propagator, $\Delta(q)$, (left panel, red continuous) and the ghost dressing function, $F(q)$, (right panel, red continuous), and the corresponding lattice data of [71]. In the right panel we also show the functions $\mathcal{C}_{1}(q)$ (blue dotted), $\mathcal{C}_{2}(q)$ (green dashed), and $\mathcal{C}_{3}(q)=F(q)$ (red continuous) given by Eq. (2.23). All functions are renormalized at $\mu=4.3 \mathrm{GeV}$.

(in our case, $\Gamma_{\mu}^{\mathrm{T}}$ ). From the perturbative point of view, several of the aforementioned issues have been studied in detail in the context of the electron propagator in QED [61], and even though the levels of technical complexity are high, they are considered to be well-understood. On the other hand, these cancellations are far more difficult to identify and enforce nonperturbatively, even if a reasonable approximation of $\Gamma_{\mu}^{\mathrm{T}}$ is furnished. Given that in the present analysis the term $\Gamma_{\mu}^{\mathrm{T}}$ is completely undetermined, and is set identically to zero, the possibility of a bona fide cancellation of the overlapping divergences is excluded from the outset.

A typical manifestation of the mismatches induced when implementing the usual simplification $Z_{c}^{-1}=1$ (or directly $\left.Z_{1}=1\right)$ is the failure of $\mathcal{M}(p)$ to display the correct anomalous dimension in the deep ultraviolet. Specifically, the asymptotic behavior of $\mathcal{M}(p)$ at one-loop is given by $[3,4,10]$

$$
\mathcal{M}_{\mathrm{UV}}(p)=\frac{C}{p^{2}}\left[\ln \left(\frac{p^{2}}{\Lambda^{2}}\right)\right]^{\gamma_{f}-1},
$$

where $C$ is a constant with mass dimension $[M]^{3}, \gamma_{f}=$ $12 /\left(11 C_{\mathrm{A}}-2 n_{f}\right)$ is the mass anomalous dimension, and $n_{f}$ is the number of active quark flavors. Instead, if the aforementioned approximation is implemented, the asymptotic behavior of the quark mass obtained from the resulting gap equation has the wrong value for $\gamma_{f}$, given by $\gamma_{f}=$ $48 /\left(35 C_{\mathrm{A}}-8 n_{f}\right)$.

A simple remedy to this problem has been put forth in [18], which is similar in spirit to an earlier proposal presented in [12]. Specifically, one carries out the substitution

$$
Z_{c}^{-1} \mathcal{K}_{\mathrm{A}, \mathrm{B}}(p, k) \rightarrow \mathcal{K}_{\mathrm{A}, \mathrm{B}}(p, k) \mathcal{C}(q),
$$

where the function $\mathcal{C}(q)$ should display the appropriate ultraviolet characteristics to convert the product

$$
\mathcal{R}(q)=\alpha_{s}(\mu) \Delta(q, \mu) F(q, \mu) \mathcal{C}(q, \mu)
$$

into a renormalization-group invariant (RGI) ( $\mu$-independent) combination, at least at one-loop.

Focusing on the function $\mathcal{C}(q)$, the requirement that $\mathcal{R}(q)$ be RGI fixes its ultraviolet behavior; specifically, for large $q^{2}$, the inverse of $\mathcal{C}(q)$ must behave as

$$
\mathcal{C}_{\mathrm{UV}}^{-1}(q)=1+\frac{9 C_{\mathrm{A}} \alpha_{s}}{48 \pi} \ln \left(\frac{q^{2}}{\mu^{2}}\right)
$$

where $C_{\mathrm{A}}$ is the eigenvalue of the Casimir operator in the adjoint representation. However, the low-energy completion of $\mathcal{C}(q)$ remains undetermined, leading to the necessity of introducing specific Ansätze for it.

The ghost dressing function $F(q)$ is the simplest quantity that fulfills (2.20) and, due to high-quality lattice simulations and extensive studies in the continuum, is quite accurately known in the entire range of Euclidean momenta. However, in the present work we will mainly focus on an alternative quantity that conforms with the aforementioned requirements, and, in addition, displays a relative enhancement with respect to $F(q)$ in the region of momenta that is particularly relevant for chiral symmetry breaking. Specifically, we will employ the so-called "ghost-gluon" mixing self-energy, denoted by $1+G(q)$, which is a crucial ingredient in contemporary application of the pinch technique [62-65], and coincides (in the Landau gauge) with the well-known Kugo-Ojima function $[41,66-69]$. The quantity $[1+G(q)]^{-1}$ has precisely the same ultraviolet behavior stated in (2.20), and SDE and lattice studies furnish its form for low and intermediate momenta (see Fig. 2); in fact, by virtue of an exact identity valid in the Landau gauge, $[1+G(0)]^{-1}=F(0)[70]$.

An accurate fit of $1+G(q)$, valid for the entire range of Euclidean momenta, is given by 
$1+G(q)=1+\frac{9 C_{\mathrm{A}} \alpha_{s}}{48 \pi} I(q) \ln \left(\frac{q^{2}+\rho_{3} m^{2}(q)}{\mu^{2}}\right)$,

with

$$
\begin{aligned}
m^{2}(q) & =\frac{m^{4}}{q^{2}+\rho_{2} m^{2}}, \\
I(q) & =1+D \exp \left(-\frac{\rho_{4} q^{2}}{\mu^{2}}\right),
\end{aligned}
$$

where $m^{2}=0.55 \mathrm{GeV}^{2}, \rho_{2}=0.60, \rho_{3}=0.50, \rho_{4}=2.08$, $\alpha_{s}=0.22, D=3.5$, and $\mu=4.3 \mathrm{GeV}$.

In the general analysis presented in the following section, we will consider three particular models for $\mathcal{C}(q)$; the first two have the function $[1+G(q)]^{-1}$ as their principal ingredient, while the third is simply $F(q)$ itself. Of course, these Ansätze are to be understood as representative cases of a wider range of qualitatively similar, but technically more involved, realizations. ${ }^{2}$

Specifically,

$$
\begin{aligned}
& \mathcal{C}_{1}(q)=[1+G(q)]^{-1}, \\
& \mathcal{C}_{2}(q)=\frac{q^{2}}{q^{2}+a_{1}}\left[1+\exp \left(-\frac{a_{2} q^{2}}{\mu^{2}}\right)\right][1+G(q)]^{-1}, \\
& \mathcal{C}_{3}(q)=F(q),
\end{aligned}
$$

where $a_{1}=0.13 \mathrm{GeV}^{2}$ and $a_{2}=50$. Note that $F^{-1}(q)$ can be also expressed by the same functional form given in Eq. (2.21), where the corresponding fitting parameters are $m^{2}=0.55 \mathrm{GeV}^{2}, \rho_{2}=2.57, \rho_{3}=0.50, \rho_{4}=3.83$, and $D=2.24$.

By construction, the three Ansätze display the same asymptotic behavior, and their perturbative tails merge into each other approximately in the region of $3 \mathrm{GeV}$ [see Fig. 2]. In addition, one can see that $\mathcal{C}_{3}(q)$ is more suppressed than $\mathcal{C}_{1}(q)$ and $\mathcal{C}_{2}(q)$ in the range of $[400 \mathrm{MeV}, 2 \mathrm{GeV}]$. On the other hand, the main difference between the first two Ansätze appears below approximately $700 \mathrm{MeV}$; thus, while $\mathcal{C}_{1}(q)$ grows monotonically and finally saturates at the value $F(0), \mathcal{C}_{2}(q)$ drops rapidly and vanishes at the origin.

Finally, carrying out the replacement given in Eq. (2.18) into Eq. (2.12), we obtain the form of the gap equation that will be used in what follows; in particular,

$$
\begin{aligned}
p^{2} A(p) & =p^{2}+4 \pi C_{\mathrm{F}} \int_{k} \mathcal{K}_{\mathrm{A}}(k, p) \mathcal{R}_{i}(q), \\
B(p) & =4 \pi C_{\mathrm{F}} \int_{k} \mathcal{K}_{\mathrm{B}}(k, p) \mathcal{R}_{i}(q),
\end{aligned}
$$

\footnotetext{
${ }^{2}$ For example, if $\mathcal{C}(q)$ originates ultimately from $\Gamma_{\mu}^{\mathrm{T}}$, it would be reasonable to expect its dependence on $k, p$, and $\theta$ to be more complicated than simply $q^{2}=(k-p)^{2}$.
}

where $\mathcal{R}_{i}(q)$ refers to the RGI product of Eq. (2.19), realized with $\mathcal{C}_{i}(q)$, for $i=1,2$ or 3 .

\section{The equations for the $X_{i}$}

The starting point in deriving the dynamical equations governing the behavior of the form factors $X_{i}$ is the diagrammatic representation of $H^{[1]}(q, k,-p)$ at the one-loop dressed approximation, shown in the second line of Fig. 1.

As we can see, the complete treatment of $H^{[1]}(q, k,-p)$ requires the previous knowledge of the full gluon-ghost vertex, $G_{\nu}$, and the complete quark-gluon vertex, $\Gamma_{\mu}$, including its transverse part. In order to reduce the level of technical complexity, we will adopt the following approximations: (i) for the full gluon-ghost vertex we simply use its tree-level value $G_{\nu}^{(0)}=(p-l)_{\mu}$, and (ii) $\Gamma_{\mu}$ is approximated by the component of $\Gamma_{\nu}^{\mathrm{BC}}$, proportional to $\gamma_{\nu}$, namely the $L_{1}^{\mathrm{FBC}}$ of Eq. (2.11) with $F(q)=1$.

With the above simplifications, one has

$$
\begin{aligned}
H^{[1]}= & 1-\frac{1}{2} i C_{\mathrm{A}} g^{2} \int_{l} \Delta^{\mu \nu}(l-k) G_{\mu}^{(0)}(p-l) \\
& \times D(l-p) S(l) L_{1}^{\mathrm{BC}}(l-k, k,-l) \gamma_{\nu} .
\end{aligned}
$$

Then, contracting the above equation with the projectors defined in Eq. (3.9) of [40], ${ }^{3}$ one obtains the following set of expressions for the individual form factors $X_{i}\left(q^{2}, k^{2}, p^{2}\right)$,

$X_{0}=1+i \pi C_{\mathrm{A}} \alpha_{s} \int_{l} \mathcal{K}(p, k, l) A(l) \mathcal{G}(k, q, l)$,

$X_{1}=i \pi C_{\mathrm{A}} \alpha_{s} \int_{l} \frac{\mathcal{K}(p, k, l) B(l)}{q^{2} h(p, k)}\left[k^{2} \mathcal{G}(p, q, l)-(p \cdot k) \mathcal{G}(k, q, l)\right]$,

$X_{2}=i \pi C_{\mathrm{A}} \alpha_{s} \int_{l} \frac{\mathcal{K}(p, k, l) B(l)}{q^{2} h(p, k)}\left[p^{2} \mathcal{G}(k, q, l)-(p \cdot k) \mathcal{G}(p, q, l)\right]$,

$X_{3}=-i \pi C_{\mathrm{A}} \alpha_{s} \int_{l} \frac{\mathcal{K}(p, k, l) A(l)}{q^{2} h(p, k)}\left[k^{2} \mathcal{G}(p, q, l)\right.$

$-(p \cdot k) \mathcal{G}(k, q, l)-\mathcal{T}(p, k, l)]$,

where we have introduced the kernel

$$
\mathcal{K}(p, k, l)=\frac{F(l-p) \Delta(l-k)[A(l)+A(k)]}{(l-p)^{2}\left[A^{2}(l) l^{2}-B^{2}(l)\right]},
$$

and the functions

$$
\begin{aligned}
\mathcal{G}(r, q, l) & =(r \cdot q)-\frac{[r \cdot(l-k)][q \cdot(l-k)]}{(l-k)^{2}}, \\
\mathcal{T}(p, k, l) & =(k \cdot q)[(p \cdot l)-(p \cdot k)]-(p \cdot q)\left[(k \cdot l)-k^{2}\right] .
\end{aligned}
$$

\footnotetext{
${ }^{3}$ Note that in the convention of momenta used in [40] we have $p_{1} \rightarrow p$ and $p_{2} \rightarrow k$.
} 
The above expressions for $X_{i}$ are expressed in Minkowski space, and depend on the three momenta $q, k$, and $p$. The Euclidean version of (2.26) is given in Eq. (3.21) of [40], and is a function of $p^{2}, k^{2}$, and the angle $\theta$ between $p$ and $k$, i.e., $X_{i}\left(p^{2}, k^{2}, \theta\right)$.

\section{NUMERICAL ANALYSIS}

In this section we present the numerical analysis and main results of the six coupled integral equations formed by $A(p), B(p)$, and the four $X_{i}$, defined by Eqs. (2.24) and (2.26), respectively.

\section{A. Inputs}

As can be observed from Eqs. (2.24) and (2.26), the numerical evaluation of $A(p), B(p)$, and $X_{i}$ requires the knowledge of three additional quantities: (i) the gluon propagator, $\Delta(q)$, (ii) the ghost dressing function, $F(q)$, and (iii) the function, $\mathcal{C}_{i}(q)$, appearing in the renormalized version of the gap equation (2.24). Ideally one could consider an even more extended system of equations, where the six equations would be coupled to the two additional SDEs that determine the momentum evolution of $\Delta(q)$ and $F(q)$; however, the resulting complexity of such an approach is very high. Instead, as was done in a series of earlier works $[18,40,72,73]$, we will employ for $\Delta(q)$ and $F(q)$ appropriate fits reconstructed from the lattice data of [71]. In the left panel of Fig. 2 we show the lattice data for $\Delta(q)$ and its corresponding fits (red continuous), renormalized at $\mu=4.3 \mathrm{GeV}$. We emphasize that these particular lattice results are "quenched", i.e., do not incorporate the effects of dynamical quark loops. In addition, on the right panel of the same figure, we show $\mathcal{C}_{1}(q)$ (blue dotted), $\mathcal{C}_{2}(q)$ (green dashed-dotted), and $\mathcal{C}_{3}(q)=F(q)$ (red continuous), all given by Eq. (2.23). Although in the deep infrared and in the intermediate region of momenta the three curves display different behaviors, one can clearly see that for values of $q \gtrsim 3 \mathrm{GeV}$ they merge into each other, as discussed in the subsection II B.

The use of quenched lattice results merits some additional clarifications, especially in view of the fact that unquenched lattice data are also available in the literature; note, for instance, that the simulations of [74] yielded results for both the gluon propagator and the ghost dressing function for $N_{f}=2$ (two degenerate light quarks), and $N_{f}=2+1+1$ (two degenerate light quarks and two heavy ones).

The main reason we refrain from using them is related with the fact that in such simulations chiral symmetry is explicitly broken due to the presence of a nonvanishing current quark mass, $m_{0}(\mu) \neq 0$, whose inclusion in the corresponding gap equation brings about nontrivial modifications. Specifically, the presence of a nonvanishing $m_{0}(\mu)$ introduces an additional term $Z_{m} m_{0}(\mu)$ on the right-hand side of the equation for $B(p)$ given in (2.12), where $Z_{m}$ is the mass renormalization constant associated with $m_{0}(\mu)$. The presence of this term complicates further the renormalization procedure of the gap equation. To see that, we recall that the renormalization conditions in the momentum subtraction scheme (MOM) require that the renormalized $A(p)$ and $B(p)$ recover their tree level values at $\mu$, i.e., $A(\mu)=1$, and $B(\mu)=m_{0}(\mu)$. Then, if one were to impose $Z_{\mathrm{F}}=1$ throughout, as was done in Sec. II B, the renormalized $A(p)$ will not recover its tree level value at the renormalization point, unless the contribution of the integral containing the kernel $\mathcal{K}_{\mathrm{A}}(k, p)$ were vanishing. Even though a "hybrid" treatment of $Z_{\mathrm{F}}$ could be adopted, ${ }^{4}$ in order to avoid these additional complications we use the quenched lattice results throughout.

Let us finally mention that, notwithstanding the aforementioned difficulties, a rough estimate of the impact of the unquenching effects in the form factors of the quark-gluon vertex in some special kinematic limits was presented in [50]; according to that analysis, the effects due to unquenching are relatively small, of the order of $10 \%$.

\section{B. Numerical results for the coupled system}

With all external inputs defined, we proceed to solve the coupled system; note in particular that the form factors $X_{i}$ will be determined for general Euclidean kinematics. Then, the vertex form factors $L_{i}$ will be obtained through direct substitution of the solutions into the Euclidean version of Eq. (2.10).

The coupled system of SDEs (2.24) and (2.26) is solved iteratively. The logarithmic grid is composed by 136 different values of momenta $p^{2}$ in the range $\left[5 \times 10^{-5} \mathrm{GeV}^{2}\right.$, $\left.5 \times 10^{3} \mathrm{GeV}^{2}\right]$, whereas the angular interval is subdivided uniformly into 25 values from 0 to $\pi$. The most costly task, the numerical evaluation of the multidimensional integrals, was tackled with an adaptive algorithm employing an 11th degree polynomial rule for the 3D integrals and a 13th degree rule for the $2 \mathrm{D}$ ones [75].

In Fig. 3 we show the numerical results for two out of the six quantities determined in our coupled system. In particular, we show the dynamical quark mass, $\mathcal{M}(p)$ (top panels), and the quark wave function, $A^{-1}(p)$ (bottom panels), obtained as solutions when we use $\mathcal{C}_{1}(q)$ in the RGI product $\mathcal{R}_{1}(q)$, defined in the Eq. (2.19). The solutions were obtained for $\alpha_{s}=0.24$ (left panels), $\alpha_{s}=$ 0.28 (center panels), and $\alpha_{s}=0.30$ (right panels).

In order to appreciate how $\mathcal{M}(p)$ and $A^{-1}(p)$ are affected by the inclusion of $H$ (or, equivalently, the $X_{i}$ ) in the construction of the quark-gluon vertex, in Fig. 3 we compare the solutions obtained with the full $\Gamma^{\mathrm{STI}}$ (blue continuous curves) with those computed using the $\Gamma_{\mu}^{\mathrm{FBC}}$ of Eq. (2.11) (orange dashed lines). Evidently, the former solutions produce higher $\mathcal{M}(p)$ compared to the latter, in

\footnotetext{
${ }^{4}$ For example, for the integral terms one may substitute $Z_{1} \mathcal{K}_{\mathrm{A}, \mathrm{B}}(p, k) \rightarrow \mathcal{K}_{\mathrm{A}, \mathrm{B}}(p, k) \mathcal{C}_{i}(q)$ as before, but treat "subtractively" the $Z_{\mathrm{F}}$ and $Z_{m}$ appearing in the "tree-level" terms.
} 

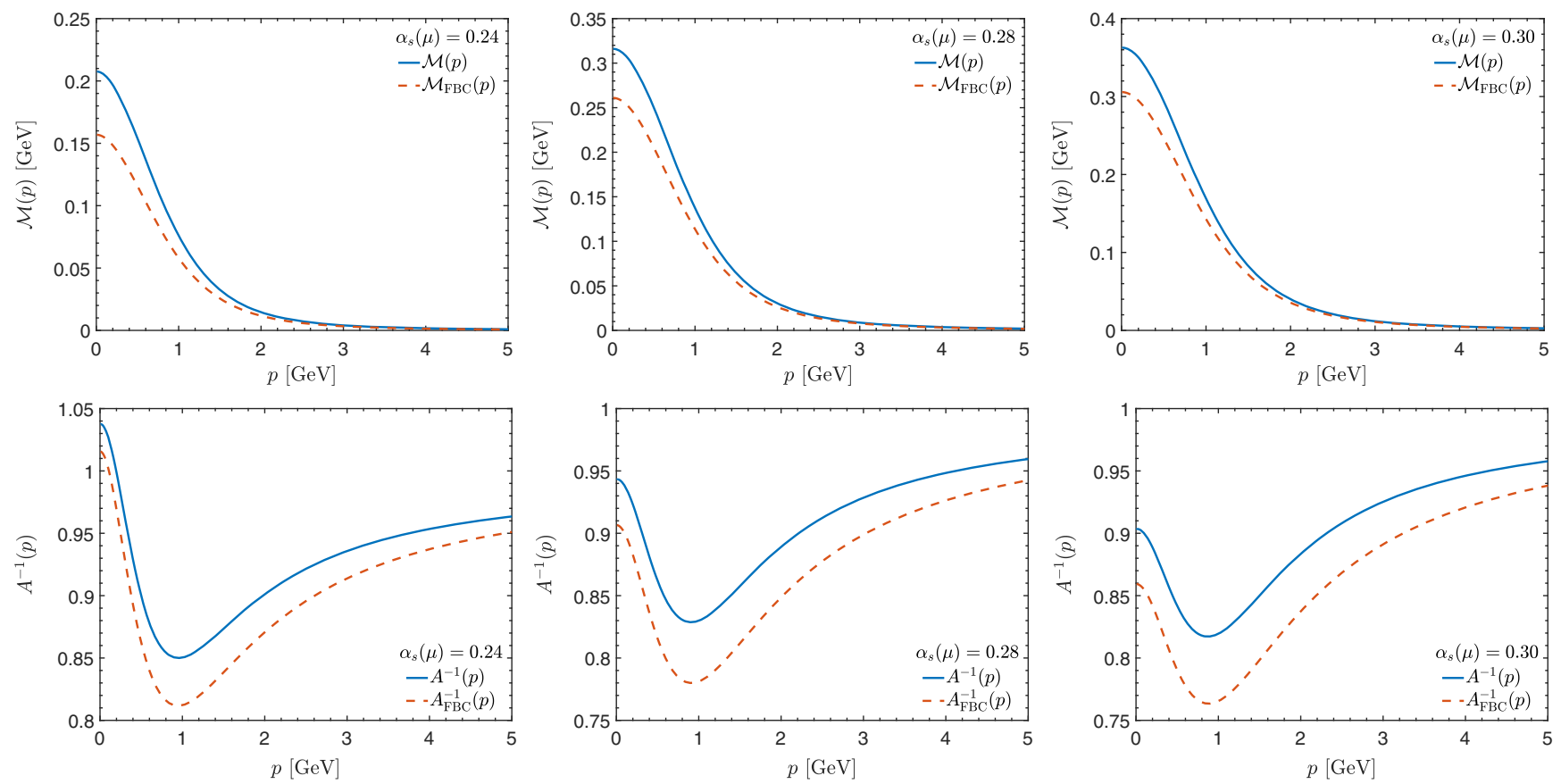

FIG. 3. Comparison of the $\mathcal{M}(p)$ (top panels) and the $A^{-1}(p)$ (bottom panels) obtained with $\Gamma_{\mu}^{\text {STI }}$ (blue continuous curve) and those obtained using $\Gamma_{\mu}^{\mathrm{FBC}}$ (orange dashed curve). All curves were obtained with the $\mathcal{C}_{1}(q)$ of Eq. (2.23), and we used $\alpha_{s}=0.24$ (left panels), $\alpha_{s}=0.28$ (central panels), and $\alpha_{s}=0.30$ (right panels).

the entire range of momentum. Of course, the quantitative difference between $\mathcal{M}(p)$ and $\mathcal{M}_{\mathrm{FBC}}(p)$ depends on the precise value of $\alpha_{s}$ : smaller values for $\alpha_{s}$ increase the difference between $\mathcal{M}(p)$ and $\mathcal{M}_{\mathrm{FBC}}(p)$. In particular, within the range of momenta between $[0,780 \mathrm{MeV}]$, we observe a difference of approximately $32 \%, 21 \%$, and $19 \%$, when $\alpha_{s}=0.24, \alpha_{s}=0.28$, and $\alpha_{s}=0.30$, respectively. A similar pattern is observed in the results for $A^{-1}(p)$ and $A_{\mathrm{FBC}}^{-1}(p)$.

The remaining four quantities are shown in the 3D plots of Fig. 4. Specifically, we present a typical set of results for the form factors $X_{i}$, where $\alpha_{s}=0.28$ and $\theta=2 \pi / 3$. We notice that all $X_{i}\left(p^{2}, k^{2}, 2 \pi / 3\right)$ are infrared finite. In addition, all curves tend asymptotically to their expected perturbative behaviors.

The $X_{i}$ computed in the previous step are subsequently fed into the Euclidean version of Eq. (2.10), thus furnishing the corresponding form factors $L_{i}$, shown in Fig. 5, where, as before, $\alpha_{s}=0.28$ and $\theta=2 \pi / 3$.

As we can see, the behavior of the $L_{i}$ (colorful surfaces) is rather similar to that obtained in Ref. [40], where $A(p)$ and $B(p)$ were treated as "external" quantities. As discussed in that work, the properties of the $L_{i}$ may be summarized as follows: (i) the four form factors are infrared finite in the entire range of momenta; (ii) the $L_{i}$ obtained indicate considerable deviations from the $L_{i}^{\mathrm{FBC}}$ represented by the cyan surface, given by Eq. (2.11); (iii) although $L_{4}$ is a non-vanishing quantity, its size is considerably suppressed for all momenta, and (iv) $L_{2}$ displays the most pronounced changes, because it is particularly sensitive to the details of the shape of $A(p)$.

Given that we have derived $L_{i}$ for general configurations, we may easily single out two special kinematics cases, namely (i) the "soft quark" limit, obtained as $p \rightarrow 0$, and (ii) the "totally symmetric" limit, where $p^{2}=k^{2}=q^{2}$ and $\theta=2 \pi / 3$. Evidently, in these limits the $L_{i}$ become functions of a single momentum, to be indicated by $r$; we will denote the corresponding form factors by $L_{i}^{q}(r)$ and $L_{i}^{\text {sym }}(r)$, respectively. In Fig. 6 we show the corresponding results, with $L_{i}^{q}(r)$ on the left panel, and $L_{i}^{\mathrm{sym}}(r)$ on the right. Note that, at the level of the 3D plots shown in Fig. 5, the $L_{i}^{\mathrm{sym}}(r)$ correspond to the "slices" defined by the planes $p=k$, where $\theta=2 \pi / 3$. In particular, $L_{4}^{\mathrm{sym}}(r)=0$. Moreover, in both cases, we recover the expected perturbative behavior for large values of the momentum $\left(L_{1}=1\right.$ and $L_{2}=L_{3}=L_{4}=0$ ).

It would be interesting to compare the above results with lattice simulations; however, the existing lattice data for the kinematic limits mentioned above are typically "contaminated" by contributions from $\Gamma_{\mu}^{\mathrm{T}}$ [45,47], due to an overall contraction by $P_{\mu \nu}(q)$ (in the Landau gauge). For the case of the "soft-gluon" configuration, $q \rightarrow 0$, a detailed comparison both with the lattice and with results found with different functional approaches has been performed in [40]. Since the present results and those of [40] are quite similar, a further comparison is of limited usefulness and will be omitted from the present work. 

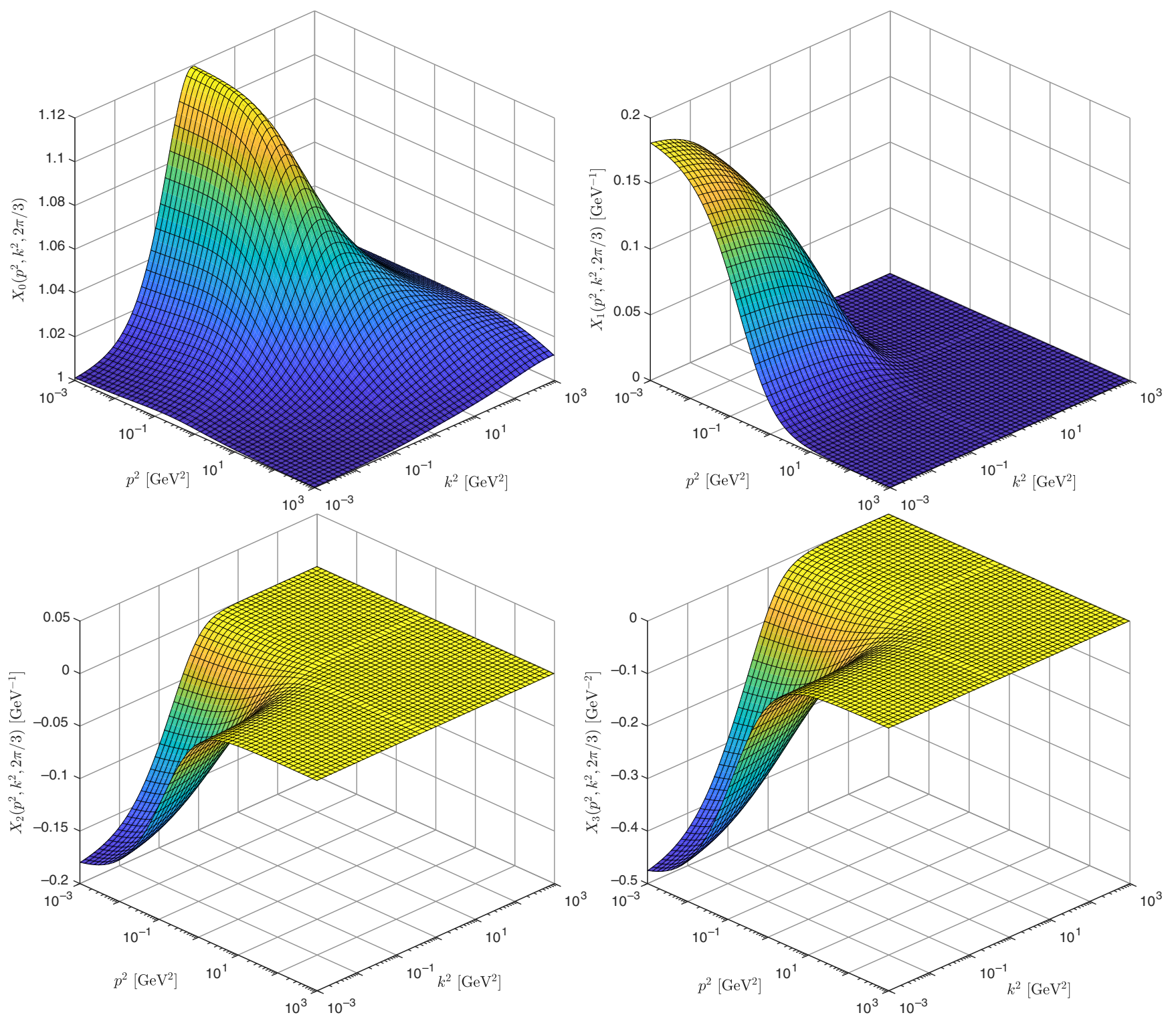

FIG. 4. The form factors $X_{i}\left(p^{2}, k^{2}, 2 \pi / 3\right)$, obtained as solution of the coupled system given by Eqs. (2.24) and (2.26), when $\alpha_{s}=0.28$ and $\theta=2 \pi / 3$.

Next, we turn our attention to the numerical impact of each individual $L_{i}$ on the value of the dynamical quark mass. The results of this exercise are presented in Fig. 7, where in both panels we show the corresponding $A^{-1}(p)$ and $\mathcal{M}(p)$, which are generated as we turn on, one by one, the form factors $L_{i}$ that compose the kernels $\mathcal{K}_{\mathrm{A}}$ and $\mathcal{K}_{\mathrm{B}}$, given by Eq. (2.13). Clearly, the largest numerical contribution comes from $L_{1}$, which is responsible for generating $54 \%$ of $\mathcal{M}(0)$. In addition, $L_{2}$ furnishes $13 \%$ of the $\mathcal{M}(0)$ value, while $L_{3}$ contributes another $23 \%$. Particularly interesting is the impact of $L_{4}$; even though it is rather suppressed [see Fig. 5], and is usually neglected in related studies $[10,12,18], L_{4}$ provides, rather unexpectedly, $10 \%$ of $\mathcal{M}(0)$.

\section{Varying the form of $\mathcal{C}(q)$}

In order to determine the influence of the functions $\mathcal{C}_{i}(q)$ on the coupled system, we repeat the analysis using $\mathcal{C}_{2}(q)$ and $\mathcal{C}_{3}(q)$ instead of $\mathcal{C}_{1}(q)$ [equivalently, $\mathcal{R}_{1}(q) \rightarrow \mathcal{R}_{2}(q)$, or $\mathcal{R}_{1}(q) \rightarrow \mathcal{R}_{3}(q)$ ].

In Fig. 8 we perform a comparative analysis of the $A^{-1}(p)$ and $\mathcal{M}(p)$ obtained when we employ the three Ansätze for $\mathcal{C}_{i}(q)$, given by Eq. (2.23), for different values of $\alpha_{s}$.

Although $\mathcal{C}_{2}(q)$ is significantly more suppressed in the deep infrared compared to $\mathcal{C}_{1}(q)$ and $\mathcal{C}_{3}(q)$ [see Fig. 2], one can observe that, essentially, the first two models generate quark masses of comparable size: the masses obtained using $\mathcal{C}_{1}(q)$ (blue dotted curve) are slightly larger than those coming from $\mathcal{C}_{2}(q)$ (green dashed curve). Clearly, the difference in the results obtained with $\mathcal{C}_{1}(q)$ and $\mathcal{C}_{2}(q)$ decreases as $\alpha_{s}$ 

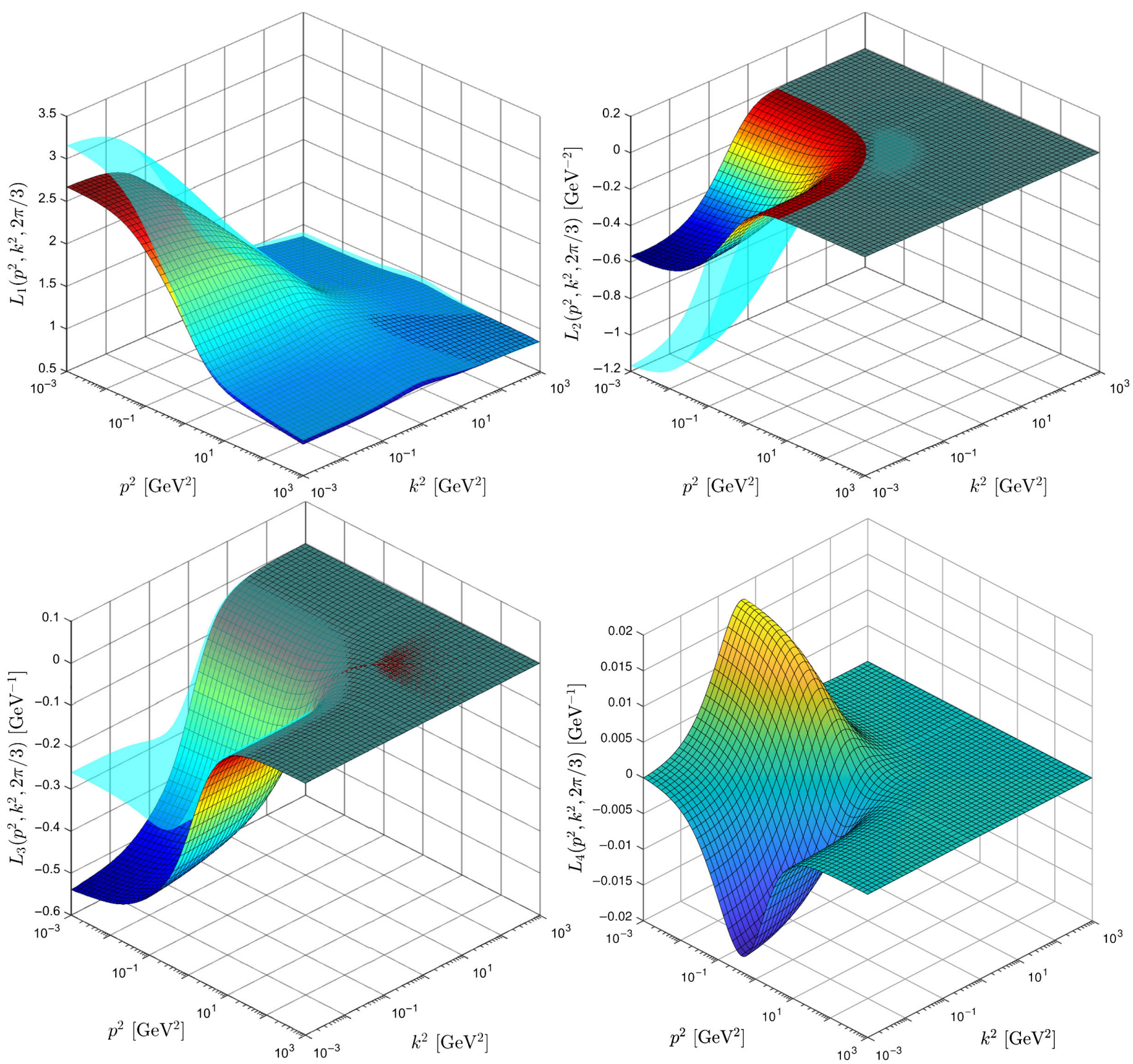

FIG. 5. The quark-gluon form factors $L_{i}$ obtained by substituting into Eq. (2.10) the solutions of the coupled system given by Eqs. (2.24) and (2.26). The results represent the case where $\alpha_{s}=0.28$ and $\theta=2 \pi / 3$.

increases; in particular, the difference between the corresponding $\mathcal{M}(0)$ computed with $\alpha_{s}=0.24, \alpha_{s}=0.28$, and $\alpha_{s}=0.30$ is about $20 \%, 10 \%$, and $6 \%$, respectively.

Instead, $\mathcal{C}_{3}(q)$ does not provide sufficient strength to the kernel of the gap equation (2.24) to trigger the onset of the dynamical mass generation, when $\alpha_{s}=0.24$ (red continuous curve in the top left panel). Although for higher values of $\alpha_{s}$ the chiral symmetry is eventually broken, one notices that the values of masses obtained are phenomenologically disfavored; specifically, one finds $160 \mathrm{MeV}$ for $\alpha_{s}=0.28$, and $217 \mathrm{MeV}$ when $\alpha_{s}=0.30$.

We emphasize that the mass pattern emerging from the above exercise is consistent with what one would expect on general grounds. Indeed, as is well-established by now, the support of the gap equation kernel in the intermediate region of momenta is crucial for the generation of phenomenologically compatible quark masses [12,18], while modifications of that kernel in the deep infrared do not affect significantly the resulting quark mass $[10,76]$. Consequently, the origin of the small difference in the $\mathcal{M}(p)$ obtained with the first two models can be naturally attributed to the slight suppression that $\mathcal{C}_{2}(q)$ displays in the region of [1-2] GeV in comparison with $\mathcal{C}_{1}(q)$, whereas the sizable suppression of $\mathcal{C}_{3}(q)$ in the range of [0.51.5] $\mathrm{GeV}$ prohibits or reduces substantially the generation of a quark mass. 

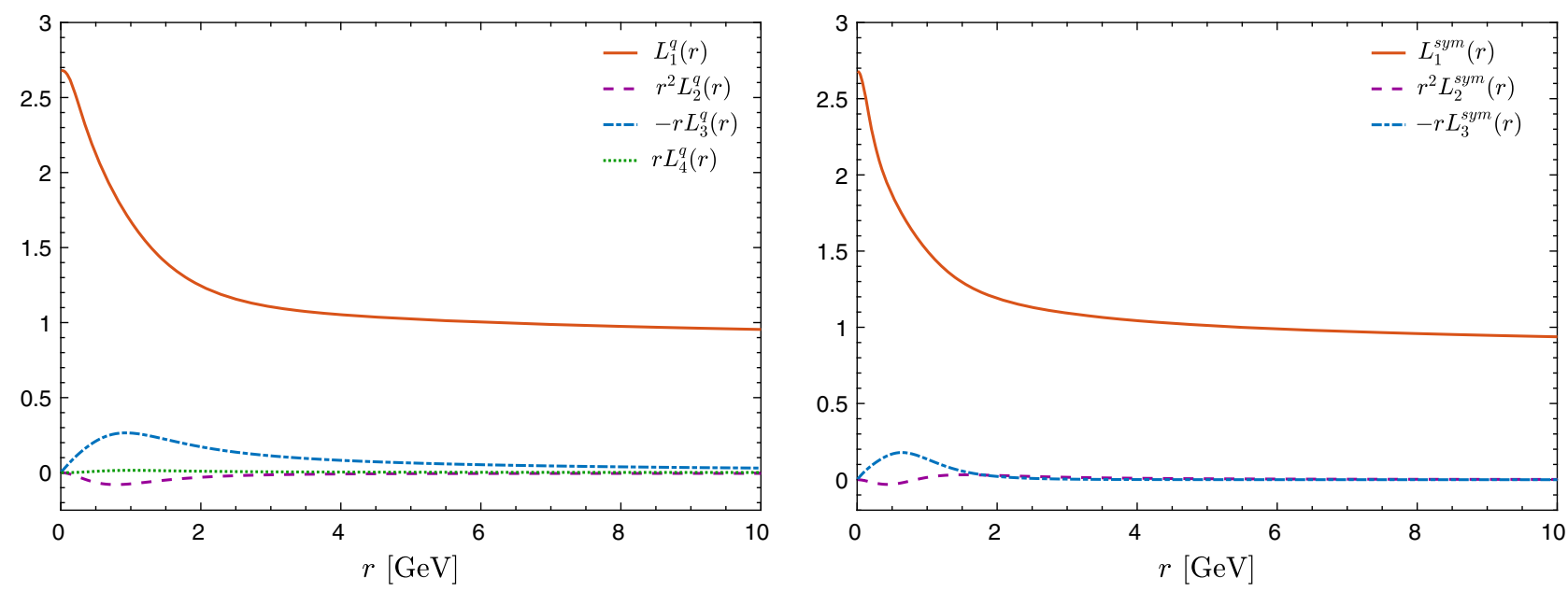

FIG. 6. The form factors $L_{i}$ for different kinematic configurations. The $L_{i}^{q}(r)$ (left panel) correspond to the soft quark configuration, while the $L_{i}^{\text {sym }}(r)$ (right panel) to the totally symmetric configuration.
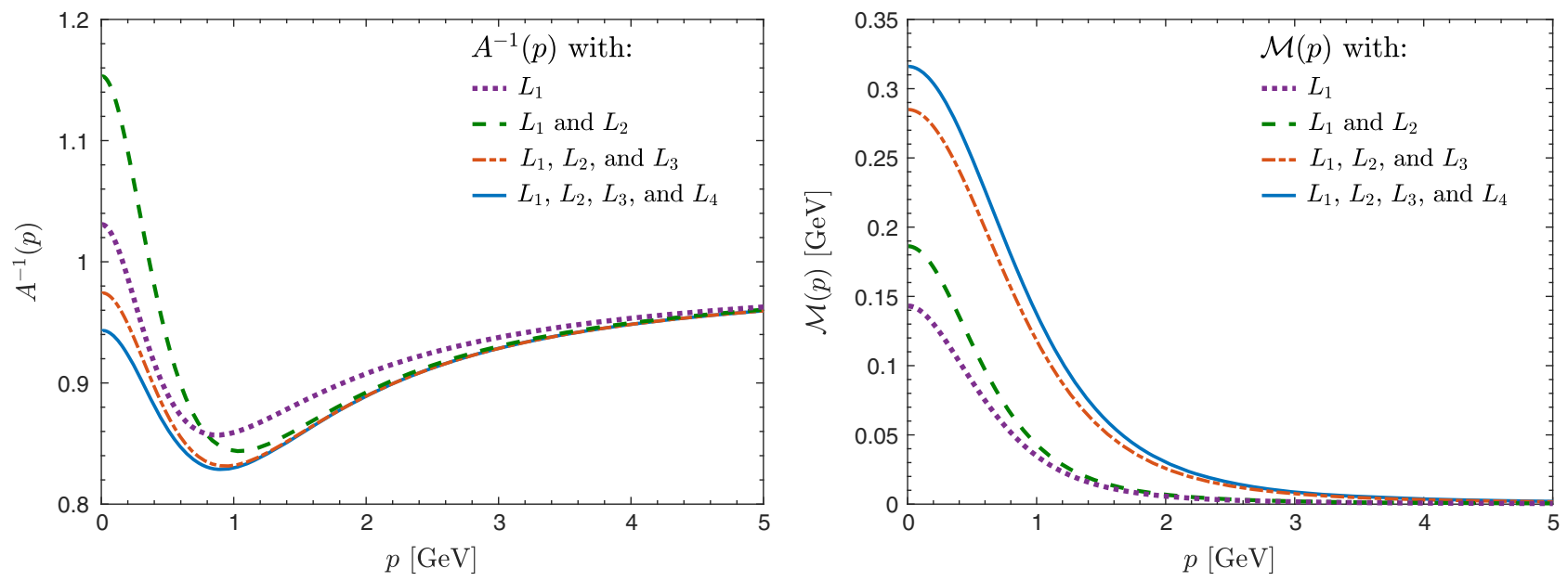

FIG. 7. Individual contributions of the quark-gluon form factors $L_{i}$ on the (i) quark wave function, $A^{-1}(p)$ (left panel), and (ii) dynamical quark mass, $\mathcal{M}(p)$ (right panel).

We conclude this subsection by presenting in Table I a detailed analysis of the impact of the scattering kernel $H$ on the dynamical mass generation, as we vary the function $\mathcal{C}_{i}(q)$. We will restrict ourselves to the comparison of the values for $\mathcal{M}_{\mathrm{FBC}}(0)$ and $\mathcal{M}(0)$; we remind the reader that, in the former case, $H$ assumes its tree-level value, while the latter is obtained from solving the system. The impact will be quantified through the relative percentage difference $I_{\mathrm{H}}=\left[\mathcal{M}(0) / \mathcal{M}_{\mathrm{FBC}}(0)-1\right] \times 100 \%$. Independently of the form that $\mathcal{C}_{i}(q)$ assumes, one notices that $I_{\mathrm{H}}$ depends on the value of $\alpha_{s}$, reaching larger values as $\alpha_{s}$ decreases. Interestingly enough, as we reach phenomenologically relevant values for $\mathcal{M}(0)$ (i.e., in the range 280$360 \mathrm{MeV}), I_{\mathrm{H}}$ practically stabilizes around $20 \%$.

\section{Fits for the constituent quark mass}

It turns out that all running quark masses $\mathcal{M}(p)$ presented in the Fig. 8 may be accurately fitted by the following physically motivated fit

$$
\mathcal{M}(p)=\frac{\mathcal{M}_{1}^{3}}{\mathcal{M}_{2}^{2}+p^{2}\left[\ln \left(p^{2}+\mathcal{M}_{3}^{2}\right) / \Lambda^{2}\right]^{1-\gamma_{f}}},
$$

where $\left(\mathcal{M}_{1}, \mathcal{M}_{2}, \mathcal{M}_{3}\right)$ are the three adjustable "mass" parameters, and $\Lambda=270 \mathrm{MeV}$.

The above formula constitutes a simple infrared completion of Eq. (2.17), where the presence of the $\mathcal{M}_{2}$ in the denominator enforces the saturation of $\mathcal{M}(p)$ at the origin, while the $\mathcal{M}_{3}$ in the argument of the logarithm improves the convergence of the fitting procedure.

It turns out that the expression

$$
\mathcal{M}(p)=\frac{\mathcal{M}_{0}}{1+\left(p^{2} / \lambda^{2}\right)^{1+d}},
$$

is yet another excellent fit for all our results for $\mathcal{M}(p)$. The functional form of Eq. (3.2) may be easier to handle when numerical integrations of $\mathcal{M}(p)$ are involved. 

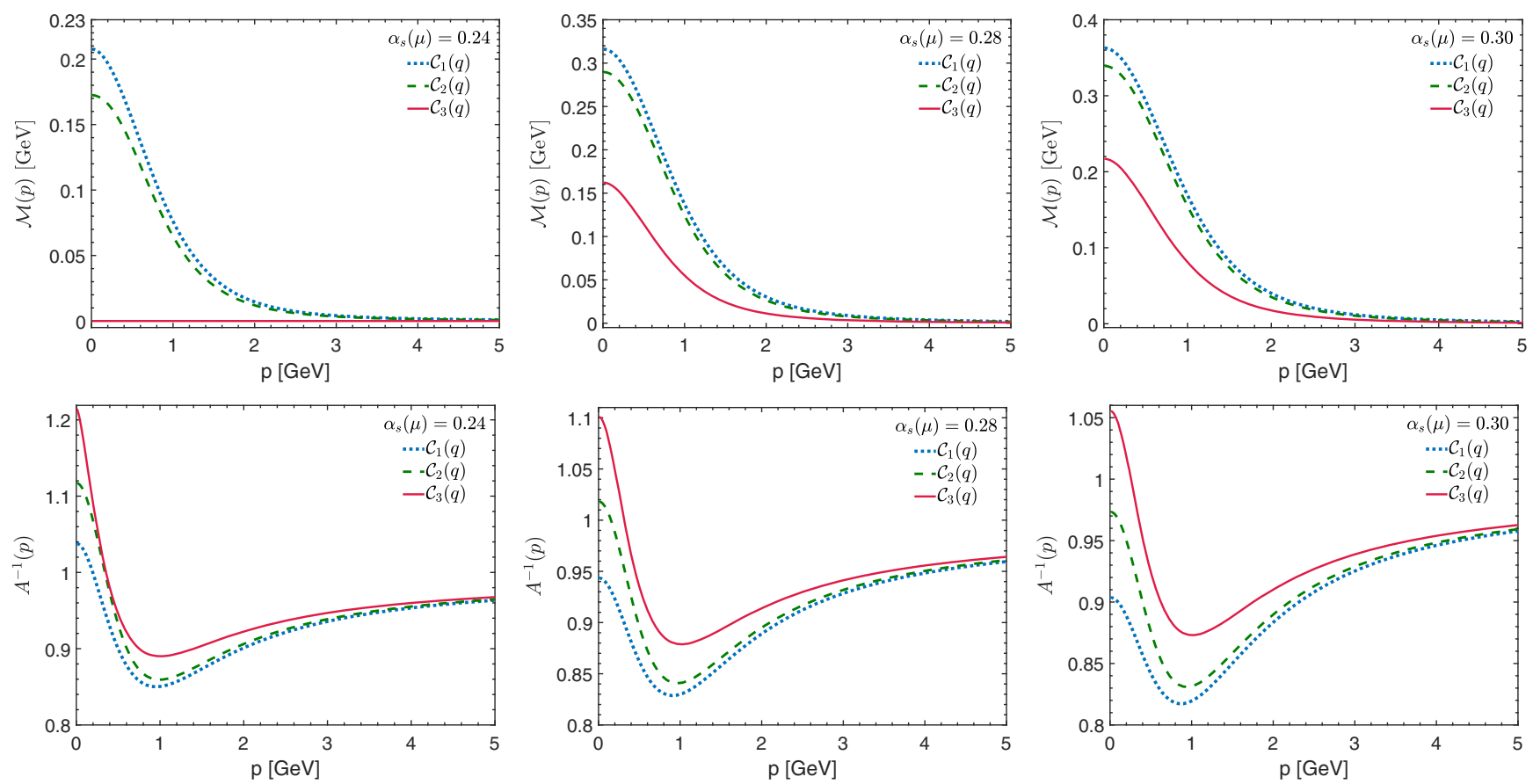

FIG. 8. Comparison of the dynamical quark masses, $\mathcal{M}(p)$, (top panels) and the quark wave function, $A^{-1}(p)$, (bottom panels) obtained when we employ the three Ansätze for $\mathcal{C}_{i}(q)$ given by Eq. (2.23) for different values of $\alpha_{s}$.

TABLE I. Comparison of the values obtained for $\mathcal{M}_{\mathrm{FBC}}(0)$ and $\mathcal{M}(0)$ when we employ the three Ansätze $\mathcal{C}_{i}(q)$ of Eq. $(2.23)$.

\begin{tabular}{|c|c|c|c|c|c|c|c|c|c|}
\hline \multirow[b]{2}{*}{$\alpha_{s}$} & \multicolumn{3}{|c|}{ Masses with $\mathcal{C}_{1}(q)[\mathrm{MeV}]$} & \multicolumn{3}{|c|}{ Masses with $\mathcal{C}_{2}(q)[\mathrm{MeV}]$} & \multicolumn{3}{|c|}{ Masses with $\mathcal{C}_{3}(q)[\mathrm{MeV}]$} \\
\hline & $\mathcal{M}_{\mathrm{FBC}}(0)$ & $\mathcal{M}(0)$ & $I_{\mathrm{H}}$ & $\mathcal{M}_{\mathrm{FBC}}(0)$ & $\mathcal{M}(0)$ & $I_{\mathrm{H}}$ & $\mathcal{M}_{\mathrm{FBC}}(0)$ & $\mathcal{M}(0)$ & $I_{\mathrm{H}}$ \\
\hline 0.24 & 157 & 207 & $32 \%$ & 114 & 172 & $51 \%$ & 0 & 0 & $0 \%$ \\
\hline 0.28 & 261 & 316 & $21 \%$ & 231 & 286 & $24 \%$ & 86 & 162 & $88 \%$ \\
\hline 0.30 & 305 & 362 & $19 \%$ & 278 & 339 & $22 \%$ & 142 & 217 & $53 \%$ \\
\hline
\end{tabular}

In Fig. 9 we superimpose the numerical solutions when $\alpha_{s}=0.24$ (red circles), $\alpha_{s}=0.28$ (purple squares), and $\alpha_{s}=0.30$ (green stars) for $\mathcal{C}_{1}(q)$ (left panel) and $\mathcal{C}_{2}(q)$ (right panel) and the fit of Eq. (3.2) (continuous curves). Since it is not possible to notice any sizable quantitative difference between the fits produced either with Eq. (3.1) or Eq. (3.2), in Fig. 9 we only show the curves for Eq. (3.2). The corresponding sets of parameters $\left(\mathcal{M}_{1}, \mathcal{M}_{2}, \mathcal{M}_{3}\right)$ and $\left(\mathcal{M}_{0}, \lambda, d\right)$ are quoted in the Table II. All fits have a reduced $\chi^{2}=0.99$.

\section{E. Estimating the pion decay constant}

In order to appreciate the impact of $H$ on a physical observable sensitive to the dynamical quark mass, we turn to the pion decay constant, $f_{\pi}$. For its computation we use an improved version of the Pagels-Stokar-Cornwall formula $[77,78]$ proposed in [79], given by. ${ }^{5}$

\footnotetext{
${ }^{5}$ The values of $f_{\pi}$ obtained from an alternative expression given in Eq. (6.27) of [10] are about $10 \%$ lower.
}

$$
\begin{aligned}
f_{\pi}^{2}= & \frac{3}{8 \pi^{2}} \int_{0}^{\infty} d y y B^{2}(y)\left\{\sigma_{V}^{2}-2\left[\sigma_{S} \sigma_{S}^{\prime}+y \sigma_{V} \sigma_{V}^{\prime}\right]\right. \\
& \left.-y\left[\sigma_{S} \sigma_{S}^{\prime \prime}-\left(\sigma_{S}^{\prime}\right)^{2}\right]-y^{2}\left[\sigma_{V} \sigma_{V}^{\prime \prime}-\left(\sigma_{V}^{\prime}\right)^{2}\right]\right\}
\end{aligned}
$$

where

$\sigma_{V}:=\frac{A(y)}{y A^{2}(y)+B^{2}(y)}, \quad \sigma_{S}:=\frac{B(y)}{y A^{2}(y)+B^{2}(y)}$.

The values quoted in the Table III for $f_{\pi}$ should be compared to the experimental value $f_{\pi}=93 \mathrm{MeV}$ [80]. Evidently, $\mathcal{C}_{3}(q)$ produces the smallest set of values for $f_{\pi}$, since the corresponding $\mathcal{M}(p)$, entering in Eq. (3.3), are quite suppressed in comparison with the others solutions obtained with $\mathcal{C}_{1}(q)$ or $\mathcal{C}_{2}(q)$. Our analysis shows clearly a preference for $\alpha_{s}$ in the range of 0.28-0.30, and for the functional forms given by $\mathcal{C}_{1}(q)$ or $\mathcal{C}_{2}(q)$. In addition, one notices that, for either $\mathcal{C}_{1}(q)$ or $\mathcal{C}_{2}(q)$, the relative percentage difference between the values for $f_{\pi}$ obtained with $\Gamma^{\mathrm{STI}}$ 

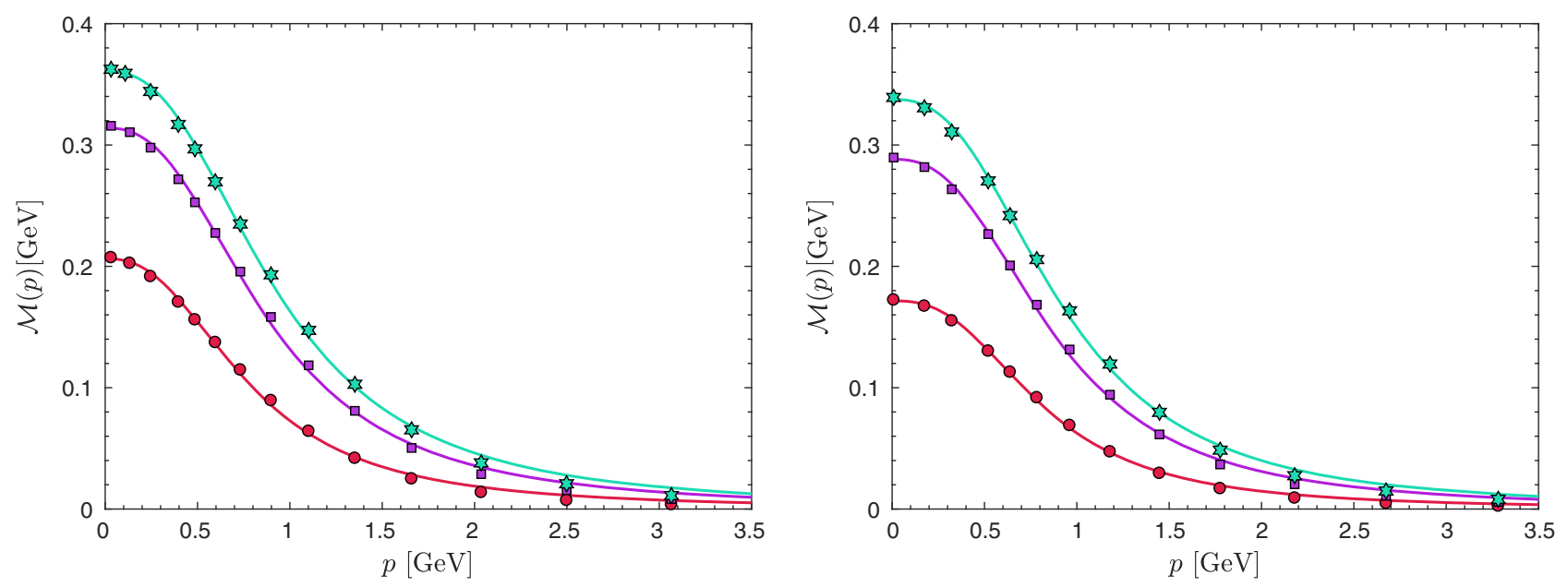

FIG. 9. The numerical solution for $\mathcal{M}(p)$ obtained using the $\mathcal{C}_{1}(q)$ (left panel) and $\mathcal{C}_{2}(q)$ (right panel). In each panel we display the solutions for $\alpha_{s}=0.24$ (red circles), $\alpha_{s}=0.28$ (purple squares) and $\alpha_{s}=0.30$ (green stars). The continuous curves represent the fit of Eq. (3.2).

and $\Gamma_{\mu}^{\mathrm{FBC}}$ are approximately $10 \%$, when $\alpha_{s}=0.28$ and $\alpha_{s}=0.30$.

\section{DISCUSSION AND CONCLUSIONS}

In this article we have performed a detailed study of the dynamical quark mass pattern that emerges when the gap equation is coupled to the four dynamical equations that

TABLE II. The sets of adjustable parameters employed for the fits given by Eqs. (3.1) and (3.2). All fits have a reduced $\chi^{2}=0.99$.

\begin{tabular}{|c|c|c|c|c|c|c|}
\hline \multirow[b]{2}{*}{$\underline{\alpha_{s}}$} & \multicolumn{3}{|c|}{ Fit given by Eq. (3.1) } & \multicolumn{3}{|c|}{ Fit given by Eq. (3.2) } \\
\hline & $\begin{array}{c}\mathcal{M}_{1} \\
{[\mathrm{MeV}]}\end{array}$ & $\begin{array}{c}\mathcal{M}_{2} \\
{[\mathrm{GeV}]} \\
\end{array}$ & $\begin{array}{c}\mathcal{M}_{3} \\
{[\mathrm{MeV}]} \\
\end{array}$ & $\begin{array}{c}\mathcal{M}_{0} \\
{[\mathrm{MeV}]} \\
\end{array}$ & $\begin{array}{c}\lambda \\
{[\mathrm{MeV}]}\end{array}$ & a \\
\hline 024 & 601 & 1.03 & 404 & 20 & 780 & 0.22 \\
\hline & 57 & & & 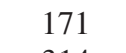 & 309 & 0.31 \\
\hline 0.28 & 758 & 1. & 426 & 314 & 878 & 0.25 \\
\hline 0.28 with $\mathcal{C}_{2}(q)$ & 715 & 1.1 & 270 & 288 & 876 & 0.28 \\
\hline 0.30 with $\mathcal{C}_{1}(q)$ & 824 & 1.25 & 358 & 361 & 925 & 0.25 \\
\hline $0.30 \mathrm{v}$ & 772 & 1.16 & 270 & 337 & 914 & 0.28 \\
\hline
\end{tabular}

TABLE III. Values for $f_{\pi}$ computed with Eq. (3.3) in [MeV]. The six sets of results were calculated using the corresponding $A(p)$ and $\mathcal{M}(p)$ obtained with the three $\mathcal{C}_{i}(q)$ given by Eq. (2.23), when we employ either the "minimal" non-abelian Ball-Chiu vertex, $\Gamma_{\mu}^{\mathrm{FBC}}$, or the complete $\Gamma^{\mathrm{STI}}$.

\begin{tabular}{|c|c|c|c|c|c|c|}
\hline \multirow[b]{2}{*}{$\alpha_{s}$} & \multicolumn{2}{|c|}{$f_{\pi}$ with $\mathcal{C}_{1}(q)$} & \multicolumn{2}{|c|}{$f_{\pi}$ with $\mathcal{C}_{2}(q)$} & \multicolumn{2}{|c|}{$f_{\pi}$ with $\mathcal{C}_{3}(q)$} \\
\hline & $\Gamma_{\mu}^{\mathrm{FBC}}$ & $\Gamma_{\mu}^{\mathrm{STI}}$ & $\Gamma_{\mu}^{\mathrm{FBC}}$ & $\Gamma_{\mu}^{\mathrm{STI}}$ & $\Gamma_{\mu}^{\mathrm{FBC}}$ & $\Gamma_{\mu}^{\mathrm{STI}}$ \\
\hline 0.24 & 62 & 73 & 52 & 67 & 0 & 0 \\
\hline 0.28 & 87 & 97 & 83 & 93 & 40 & 61 \\
\hline 0.30 & 97 & 107 & 93 & 103 & 57 & 75 \\
\hline
\end{tabular}

determine the structure of the quark-ghost kernel, $H$, and, in turn, the STI-saturating part of the quark-gluon vertex, $\Gamma_{\mu}$. The analysis has been carried out in the Ball-Chiu tensorial basis, and the dynamical equations for $H$ are derived within the one-loop dressed truncation scheme, under certain simplifying assumptions for the vertices appearing in them. The corresponding gap equation that generates the dynamical quark mass has been treated in the chiral limit (vanishing "current" mass).

The numerical effect of including a nontrivial $H$ into the construction of the $\Gamma_{\mu}$ that enters in the gap equation is rather sizable. Indeed, as we have seen in the Table I, while its precise contribution depends on the value of $\alpha_{s}$, it accounts for approximately $20 \%$ of the dynamical quark mass generated, when $\mathcal{M}(0)$ is in the range of 280-360 MeV.

The impact of $H$ on the dynamics of chiral symmetry breaking was also estimated indirectly, through the determination of the pion decay constant, $f_{\pi}$. When phenomenological compatible quark masses are generated, we see that the inclusion of $H$ into $\Gamma_{\mu}$, i.e., the transition $\Gamma_{\mu}^{\mathrm{BC}} \rightarrow \Gamma_{\mu}$, amounts to a $10 \%$ increase in the value of $f_{\pi}$.

It is important to emphasize that in the present analysis a nontrivial structure of the vertex form factor $L_{4}$ was included in the gap equation. Despite the fact that $L_{4}$ is rather suppressed compared to $L_{1}, L_{2}, L_{3}$, as shown in the Fig. 5, our findings indicate that it accounts for $10 \%$ of total $\mathcal{M}(0)$ generated. Therefore, $L_{4}$ contributes to the dynamical mass generation practically with the same strength as $L_{2}$. This result, in turn, seems to suggest that $L_{4}$ provides a more "focused" support to the gap equation kernel, enhancing it precisely in the range of momenta that drive the onset of chiral symmetry breaking. To the best of our knowledge, such a concrete quantitative statement on the impact of $L_{4}$ appears for the first time in the literature. 
Given that the multiplicative renormalizability of the quark propagator constitutes a notoriously difficult task, the restoration of the correct one-loop anomalous dimension for $\mathcal{M}(p)$ has been accomplished through the introduction ("by hand") of a set of functions, $\mathcal{C}_{i}(q)$, which in the deep ultraviolet display the required asymptotic behavior, but differ substantially at the level of their infrared "completion." The support of $\mathcal{C}_{i}(q)$ in the region of $[500 \mathrm{MeV}$, $1.5 \mathrm{GeV}]$ is crucial for the generation of quark masses of the order of $300 \mathrm{MeV}$. In fact, any suppression in the behavior of $\mathcal{C}_{i}(q)$, as reported in the case $\mathcal{C}_{3}(q)$ given by Eq. (2.23), can diminish or even eradicate the desired phenomenon.

The difficulties in enforcing multiplicative renormalizability at the level of the gap equation, as mentioned above, make the study of the transverse part of the quark-gluon vertex all the more pressing. Even though the relevance of $\Gamma_{\mu}^{\mathrm{T}}$ in this context has been amply emphasized, and various techniques have been put forth for restricting its structure [50,81-85], a well-defined framework for its systematic determination still eludes us. In particular, it would be rather important to obtain reliable results for $\Gamma_{\mu}^{\mathrm{T}}$ by means of nonperturbative methods in the continuum (e.g., SDEs $[10,13,41,62]$ or functional renormalization group [86]), especially in view of its theoretical and numerical relevance for chiral symmetry breaking.

As mentioned in the Introduction, we have carried out a "quenched" calculation, given that the gluon and ghost propagators used as inputs for solving the system of integral equations are obtained from lattice simulations with no dynamical quarks [71]. To be sure, a more complete analysis ought to take unquenching effects into account; their inclusion is expected to affect the results mainly due to the modifications induced to the gluon propagator (see, e.g., [74] for unquenched lattice results, and $[29,38,52-54]$ for related studies). A preliminary study presented in [50] indicates a slight increase, of the order $6 \%-10 \%$, in the form factors of the quark-gluon vertex, evaluated in some special kinematic limits. Of course, a complete study needs be carried out in order to determine if such an increase persists at the level of the coupled system, and the changes that it might induce to the gap equation and the quark mass derived from it.

\section{ACKNOWLEDGMENTS}

The research of J.P. is supported by the Spanish Ministerio de Economía y Competitividad (MEYC) under Grants No. FPA2014-53631-C2-1-P and No. SEV-20140398, and Generalitat Valenciana under Grant Prometeo II/ 2014/066. The work of A. C. A, J. C. C., and M. N. F. are supported by the Brazilian National Council for Scientific and Technological Development (CNPq) under the Grants No. 305815/2015, No. 141981/2013-0, and No. 142226/ 2016-5, respectively. A. C. A also acknowledges the financial support from São Paulo Research Foundation (FAPESP) through the projects No. 2017/07595-0 and No. 2017/05685-2. This research was performed using the Feynman Cluster of the John David Rogers Computation Center (CCJDR) in the Institute of Physics "Gleb Wataghin," University of Campinas.
[1] Y. Nambu and G. Jona-Lasinio, Phys. Rev. 122, 345 (1961).

[2] K. D. Lane, Phys. Rev. D 10, 2605 (1974).

[3] H. D. Politzer, Nucl. Phys. B117, 397 (1976).

[4] V. A. Miransky and P. I. Fomin, Phys. Lett. 105B, 387 (1981).

[5] D. Atkinson and P. W. Johnson, Phys. Rev. D 37, 2296 (1988).

[6] N. Brown and M. R. Pennington, Phys. Rev. D 38, 2266 (1988).

[7] A. G. Williams, G. Krein, and C. D. Roberts, Ann. Phys. (N.Y.) 210, 464 (1991).

[8] J. Papavassiliou and J. M. Cornwall, Phys. Rev. D 44, 1285 (1991).

[9] F. T. Hawes, C. D. Roberts, and A. G. Williams, Phys. Rev. D 49, 4683 (1994).

[10] C. D. Roberts and A. G. Williams, Prog. Part. Nucl. Phys. 33, 477 (1994).

[11] A. A. Natale and P. S. Rodrigues da Silva, Phys. Lett. B 392, 444 (1997).

[12] C. S. Fischer and R. Alkofer, Phys. Rev. D 67, 094020 (2003).
[13] P. Maris and C. D. Roberts, Int. J. Mod. Phys. E 12, 297 (2003).

[14] A. C. Aguilar, A. Nesterenko, and J. Papavassiliou, J. Phys. G 31, 997 (2005).

[15] P. O. Bowman, U. M. Heller, D. B. Leinweber, M. B. Parappilly, A. G. Williams, and J.-b. Zhang, Phys. Rev. D 71, 054507 (2005).

[16] V. Sauli, J. Adam, Jr., and P. Bicudo, Phys. Rev. D 75, 087701 (2007).

[17] J. M. Cornwall, arXiv:0812.0359.

[18] A. C. Aguilar and J. Papavassiliou, Phys. Rev. D 83, 014013 (2011).

[19] I. C. Cloet and C. D. Roberts, Prog. Part. Nucl. Phys. 77, 1 (2014).

[20] M. Mitter, J. M. Pawlowski, and N. Strodthoff, Phys. Rev. D 91, 054035 (2015).

[21] J. Braun, L. Fister, J. M. Pawlowski, and F. Rennecke, Phys. Rev. D 94, 034016 (2016).

[22] W. Heupel, T. Goecke, and C. S. Fischer, Eur. Phys. J. A 50, 85 (2014). 
[23] D. Binosi, L. Chang, J. Papavassiliou, S.-X. Qin, and C. D. Roberts, Phys. Rev. D 95, 031501 (2017).

[24] J. S. Ball and T.-W. Chiu, Phys. Rev. D 22, 2542 (1980).

[25] A. Kizilersu, M. Reenders, and M. R. Pennington, Phys. Rev. D 52, 1242 (1995).

[26] A. I. Davydychev, P. Osland, and L. Saks, Phys. Rev. D 63, 014022 (2000).

[27] R. Bermudez, L. Albino, L. X. Gutierrez-Guerrero, M. E. Tejeda-Yeomans, and A. Bashir, Phys. Rev. D 95, 034041 (2017).

[28] A. Bender, C. D. Roberts, and L. Von Smekal, Phys. Lett. B 380, 7 (1996).

[29] M. S. Bhagwat and P. C. Tandy, Phys. Rev. D 70, 094039 (2004).

[30] F. J. Llanes-Estrada, C. S. Fischer, and R. Alkofer, Nucl. Phys. B, Proc. Suppl. 152, 43 (2006).

[31] A. Holl, A. Krassnigg, and C. D. Roberts, Nucl. Phys. B, Proc. Suppl. 141, 47 (2005).

[32] H. H. Matevosyan, A. W. Thomas, and P. C. Tandy, Phys. Rev. C 75, 045201 (2007).

[33] R. Alkofer, C. S. Fischer, F. J. Llanes-Estrada, and K. Schwenzer, Ann. Phys. (Amsterdam) 324, 106 (2009).

[34] M. Hopfer, A. Windisch, and R. Alkofer, Proc. Sci. ConfinementX2012 (2012) 073 [arXiv:1301.3672].

[35] A. C. Aguilar, D. Binosi, J. Cardona, and J. Papavassiliou, Proc. Sci. ConfinementX2012 (2012) 103 [arXiv:1301.4057].

[36] E. Rojas, J. de Melo, B. El-Bennich, O. Oliveira, and T. Frederico, J. High Energy Phys. 10 (2013) 193.

[37] R. Williams, Eur. Phys. J. A 51, 57 (2015).

[38] R. Williams, C. S. Fischer, and W. Heupel, Phys. Rev. D 93, 034026 (2016).

[39] H. Sanchis-Alepuz and R. Williams, Phys. Lett. B 749, 592 (2015).

[40] A. C. Aguilar, J. C. Cardona, M. N. Ferreira, and J. Papavassiliou, Phys. Rev. D 96, 014029 (2017).

[41] C. S. Fischer, J. Phys. G 32, R253 (2006).

[42] J. Skullerud, P. O. Bowman, and A. Kizilersu, arXiv:hep-lat/ 0212011.

[43] J. Skullerud and A. Kizilersu, J. High Energy Phys. 09 (2002) 013.

[44] J. I. Skullerud, P. O. Bowman, A. Kizilersu, D. B. Leinweber, and A. G. Williams, J. High Energy Phys. 04 (2003) 047.

[45] J. I. Skullerud, P. O. Bowman, A. Kizilersu, D. B. Leinweber, and A. G. Williams, Nucl. Phys. B, Proc. Suppl. 141, 244 (2005).

[46] H.-W. Lin, Phys. Rev. D 73, 094511 (2006).

[47] A. Kizilersu, D. B. Leinweber, J.-I. Skullerud, and A. G. Williams, Eur. Phys. J. C 50, 871 (2007).

[48] O. Oliveira, A. Kizilersu, P. J. Silva, J.-I. Skullerud, A. Sternbeck, and A. G. Williams, Acta Phys. Pol. B Proc. Suppl. 9, 363 (2016).

[49] A. Sternbeck, P.-H. Balduf, A. Kizilersu, O. Oliveira, P. J. Silva, J.-I. Skullerud, and A. G. Williams, Proc. Sci. LATTICE2016 (2017) 349 [arXiv:1702.00612].

[50] A. C. Aguilar, D. Binosi, D. Ibañez, and J. Papavassiliou, Phys. Rev. D 90, 065027 (2014).

[51] C. S. Fischer, R. Alkofer, W. Cassing, F. J. Llanes-Estrada, and P. Watson, Nucl. Phys. B, Proc. Suppl. 153, 90 (2006).
[52] C. S. Fischer, P. Watson, and W. Cassing, Phys. Rev. D 72, 094025 (2005).

[53] A. K. Cyrol, M. Mitter, J. M. Pawlowski, and N. Strodthoff, Phys. Rev. D 97, 054006 (2018).

[54] A. C. Aguilar, D. Binosi, and J. Papavassiliou, Phys. Rev. D 86, 014032 (2012).

[55] O. Nachtmann and W. Wetzel, Nucl. Phys. B187, 333 (1981).

[56] N. Brown and N. Dorey, Mod. Phys. Lett. A 06, 317 (1991).

[57] D. C. Curtis and M. R. Pennington, Phys. Rev. D 42, 4165 (1990).

[58] D. C. Curtis and M. R. Pennington, Phys. Rev. D 48, 4933 (1993).

[59] J. C. R. Bloch, Phys. Rev. D 64, 116011 (2001).

[60] J. C. R. Bloch, Phys. Rev. D 66, 034032 (2002).

[61] A. Kizilersu and M. R. Pennington, Phys. Rev. D 79, 125020 (2009).

[62] D. Binosi and J. Papavassiliou, Phys. Rep. 479, 1 (2009).

[63] A. C. Aguilar, D. Binosi, and J. Papavassiliou, Phys. Rev. D 78, 025010 (2008).

[64] D. Binosi, L. Chang, J. Papavassiliou, and C. D. Roberts, Phys. Lett. B 742, 183 (2015).

[65] D. Binosi, C. Mezrag, J. Papavassiliou, C. D. Roberts, and J. Rodriguez-Quintero, Phys. Rev. D 96, 054026 (2017).

[66] T. Kugo and I. Ojima, Prog. Theor. Phys. Suppl. 66, 1 (1979).

[67] T. Kugo, arXiv:hep-th/9511033.

[68] P. A. Grassi, T. Hurth, and A. Quadri, Phys. Rev. D 70, 105014 (2004).

[69] A. C. Aguilar, D. Binosi, and J. Papavassiliou, J. High Energy Phys. 11 (2009) 066.

[70] A. C. Aguilar, D. Binosi, J. Papavassiliou, and J. RodriguezQuintero, Phys. Rev. D 80, 085018 (2009).

[71] I. L. Bogolubsky, E. M. Ilgenfritz, M. Muller-Preussker, and A. Sternbeck, Proc. Sci. LATTICE2007 (2007) 290 [arXiv:0710.1968].

[72] A. C. Aguilar, D. Binosi, and J. Papavassiliou, Phys. Rev. D 84, 085026 (2011).

[73] A. C. Aguilar, D. Binosi, and J. Papavassiliou, J. High Energy Phys. 01 (2012) 050.

[74] A. Ayala, A. Bashir, D. Binosi, M. Cristoforetti, and J. Rodriguez-Quintero, Phys. Rev. D 86, 074512 (2012).

[75] J. Berntsen, T. O. Espelid, and A. Genz, ACM Trans. Math. Softw. 17, 452 (1991).

[76] P. Maris and P. C. Tandy, Phys. Rev. C 60, 055214 (1999).

[77] H. Pagels and S. Stokar, Phys. Rev. D 20, 2947 (1979).

[78] J. M. Cornwall, Phys. Rev. D 22, 1452 (1980).

[79] C. D. Roberts, Nucl. Phys. A605, 475 (1996).

[80] C. Patrignani et al. (Particle Data Group), Chin. Phys. C 40, 100001 (2016).

[81] K.-I. Kondo, Int. J. Mod. Phys. A 12, 5651 (1997).

[82] H.-X. He, F. Khanna, and Y. Takahashi, Phys. Lett. B 480, 222 (2000).

[83] H.-X. He, Commun. Theor. Phys. 46, 109 (2006).

[84] H.-X. He, Int. J. Mod. Phys. A 22, 2119 (2007).

[85] S.-X. Qin, L. Chang, Y.-X. Liu, C. D. Roberts, and S. M. Schmidt, Phys. Lett. B 722, 384 (2013).

[86] J. M. Pawlowski, Ann. Phys. (Amsterdam) 322, 2831 (2007). 